\title{
Helvetismen: Nationale und areale Varianten? Kodifizierung und sprachliche Realität. ${ }^{1}$
}

\author{
BRITTA JUSKA-BACHER
}

Abstract

The concept of pluricentricity - introduced by Michael Clyne in its modern form - is widely accepted for the German language area. It states that German is an official language in three nations - Germany, Switzerland and Austria - where it has evolved into discrete standard varieties. This is also reflected in lexicography, e.g. in the Variantenwörterbuch des Deutschen, which lists national lexical variants from the three countries.

Despite agreement on pluricentricity, there is controversy among linguists about what determines geographical boundaries between language varieties, in particular about the relative importance of national or dialect areas such as Bavarian and Alemannic, either of which transcend the borders between two and three countries (Austria/South-East Germany, and Switzerland/Eastern Austria/South-West Germany). Among the linguists who have tried to resolve the controversy, Ingo Reiffenstein emphasises the overlap of national and areal variation.

This article addresses the co-existence of national and dialect-area influences. It investigates the spatial distribution of familiarity with a set of phrasemes which relevant contemporary dictionaries have defined as Helvetisms.

A survey of 1,000 individuals from Switzerland, Germany and Austria examined their familiarity with 18 alleged phraseological Helvetisms; participants were also asked to guess at the phrasemes' spatial distribution. On the basis of this empirical data, we sought to confirm whether the phrasemes are in fact restricted to Switzerland, or whether they occur in (parts of) the other standard variety areas, paying particular attention to areas adjacent to Switzerland, i.e. Austria and Baden-Württemberg in Germany.

The definition of a (phraseological) Helvetism implies two main assumptions: firstly, the phraseme needs to be part of this standard variety of German, expressed by its codification in dictionaries, with Koller also requiring 
that speakers be aware of the phraseme being a Swiss variant. Secondly, the phraseme needs to be widely used in German-speaking Switzerland, and only here.

Three criteria - e.g. existing dictionaries, expert opinion, and empirical data (corpus linguistics or survey data) - indicate codification of phrasemes in dictionaries. While the lexicographers of three dictionaries relevant for national phraseme variants, i.e. Duden Redewendungen (Dudenredaktion 2008), Schweizer Wörterbuch (Meyer 2006), and Variantenwörterbuch (Ammon et al. 2004), refer to all three of these criteria, including empirical data collected from corpora, none have ascertained whether informants from the relevant regions are familiar with the phrasemes. Hence, the three dictionaries can establish only an approximate relation between relevant phrasemes and national language areas; none of them contain reliable data on the spatial distribution of phraseological units, particularly in crossborder dialect areas. Another problem in dealing with phraseological Helvetisms is that of terminological imprecision due to insufficient knowledge of spatial distribution; phrasemes known to be in use across the entire Alemannic area (German and Austrian border areas with Switzerland) are nevertheless defined as Helvetisms in contemporary dictionaries. A third problem is our limited knowledge of speaker awareness. Several studies have found evidence for low awareness by Swiss speakers of their national variety and its variants, which was one reason for Koller to entirely negate the existence of a Swiss national variety.

\section{The survey}

We conducted a survey on the knowledge of a group of 18 phrasemes codified as Helvetisms in the above-mentioned dictionaries. Almost 1,000 informants from Austria, Germany and Switzerland (the largest cohort) answered multiple-choice questions regarding their familiarity with the phrasemes, and the form or meaning of phrasemes; participants were also asked to guess at the spatial distribution of the phrasemes in German-speaking countries, and to supply personal information on age, gender, education and place of residence. We used the data to create maps showing the spatial distribution of these supposed Helvetisms.

\section{Results}

1. The data collected from Swiss, German and Austrian speakers regarding their familiarity with so-called phraseological Helvetisms show both national and areal influences, and therefore confirm the co-existence of national and areal concepts. Three groups of phrasemes were distinguished according to 
their spatial distribution, with the first group restricted to but widespread within the Swiss language area (e.g. dastehen wie der Esel am Berg - literally: 'to stand like a donkey at the foot of the mountain', English equivalent: 'to look like a [dying] duck in a thunderstorm'), and therefore Helvetisms sensu stricto. A second group of phrasemes (e.g. durchs Band weg - literally: 'off through the band', meaning 'every single one') was known outside Switzerland to relevant degrees (i.e. $>40 \%$ of participants), which means that, taking their actual spatial distribution into account, they can be named more appropriately, e.g. Austro-Helvetism (known in Switzerland and Austria), Alemannism (well-known throughout the Allemannic dialect region), etc. The third group of phrasemes was re-stricted to some regions of Switzerland but unknown in the other German speaking areas (e.g. das Feuer im Elsass sehen - literally: 'to see the fire in Alsace', meaning 'to be in great pain'), and may therefore be considered regional Helvetisms or Swiss regionalisms to indicate their restricted distribution in Switzerland.

2. The empirical data give an impression of Swiss speakers' awareness of their national variants. Average awareness of a phraseme's status as a Helvetism was $50 \%$, which means one in two Swiss informants correctly identified a Helvetism - a comparatively low value that confirms outcomes of other studies. However, these awareness values should be interpreted with caution since classification as a Helvetism may be influenced by several factors, e.g. correct classification was highly correlated with familiarity of the phrasemes; well-known phrasemes were more often correctly classified as Helvetisms.

Moreover, our findings suggest that even relatively low awareness need not be a criterion for the general denial of a standard variety, as Koller does for Switzerland.

Linguists can and should support speakers' awareness of the national variety, and reinforce their linguistic self-confidence in the non-dominant language community. To use a Swiss example, the codification of national variants can demonstrate that common French loan words for Bürgersteig, Hähnchen, Fahrrad - i.e. Trottoir, Poulet, Velo (pavement, chicken, bicycle) are fully equivalent to their German counterparts.

\section{Plurizentrik der deutschen Sprache: Plurinationalität oder Pluriarealität?}

Grundlage der heutigen Plurizentrikdiskussion ${ }^{2}$ stellen die Ausführungen von Clyne dar (1984, siehe auch 1992), in denen das Deutsche als 
plurizentrische, im Sinne einer plurinationalen Sprache mit den drei Vollzentren Deutschland, Österreich und der Schweiz beschrieben wird. ${ }^{3}$ In diesen Ländern haben sich aufgrund der unterschiedlichen nationalen Entwicklung je eigene standardsprachliche Varietäten herausgebildet. Dies Konzept der nationalen Varietäten ist prinzipiell akzeptiert und belegt, wovon u.a. verschiedene Wörterbücher zeugen, die sich die Erfassung der Lexik dieser Varietäten zum Ziel gesetzt haben: Das Variantenwörterbuch des Deutschen von Ammon et al. (2004), Wie sagt man in Österreich? von Ebner (2009) und das Schweizer Wörterbuch von Meyer (2006), Neuauflage von Wie sagt man in der Schweiz?, Meyer (1989).

Die zentrale Gewichtung des nationalen Einflusses ist allerdings verschiedentlich auch auf Kritik gestoßen. So wurde argumentiert, dass die räumliche Komponente einerseits nur eine von zahlreichen sozialen Dimensionen darstelle (siehe beispielsweise Koller 1999: 156) und dass andererseits bei der räumlichen Gliederung des deutschen Sprachraums die areale Komponente (sprich der Einfluss der Dialekträume auf die Standardsprache dieser Gebiete) schwerer gewichte als die nationale (Scharloth 2005: 237). ${ }^{4}$ Es wurde vorgeschlagen, die Begriffe „Plurizentrik“ und „Plurinationalität“ durch „Pluriarealität“ zu ersetzen beziehungsweise zu konkretisieren (Ammon 1995: 49; Scheuringer 1997: 339ff). Diesem Gedanken widerspricht wiederum von Polenz (1999: 126), der auf den Abbau älterer Dialekt- und Regionalkontinua, die Scheuringer (1990) für das bairische Dialektkontinuum (Deutschland und Österreich) sowie Schifferle (1995) und Seidelmann (1983) für das alemannischen Dialektkontinuum (Deutschland und die Schweiz) aufgezeigt haben, und auf die zunehmende soziolinguistische Bedeutung der heutigen Staatsgrenzen hinweist. Eine Synthese der Positionen in der Plurinationalitäts- vs. Pluriarealität-Diskussion versucht Reiffenstein (2001: 88; siehe auch Hofer 2003: 479), indem er das Nebeneinander beider Konzepte herausstellt, dahingehend dass nationale Varietät und regionale Variation sich überlappen.

In Abbildung 1 wird diese Situation vereinfacht grafisch dargestellt. Dabei symbolisieren die durchgezogenen Linien die nationalen Grenzen und die von ihnen eingeschlossenen Flächen die nationalen Varietäten, d.h. das deutsche, Deutschschweizer und österreichische Vollzentrum. Die gestrichelten Linien umgeben den alemannischen Dialektraum (die areale alemannische Varietät), die gepunkteten Linien den bairischen Dialektraum (die areale bairische Varietät). Der Terminus areale Verbreitung bezeichnet im Folgenden eine Verbreitung innerhalb des alemannischen beziehungsweise barischen Dialektraums (zur räumlichen Erstreckung dieser Areale siehe Kapitel 2). 


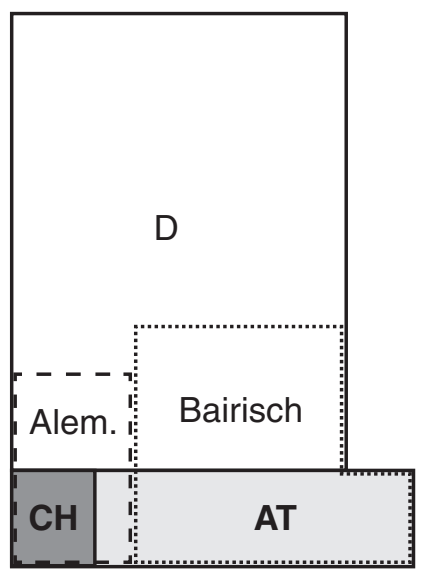

Abbildung 1. Das Deutsche als plurinationale und pluriareale Sprache Innerhalb der durchgezogenen Linien in verschiedenen Graustufen: die nationalen Varietäten $(D=$ Deutschland, $C H=$ Deutschschweiz, $A T=$ Österreich). Die gestrichelten Linien markieren den alemannischen, die gepunkteten Linien den bairischen Dialektraum.

Im vorliegenden Artikel wird exemplarisch anhand einiger Phraseme, die in aktuellen Wörterbüchern der Schweizer nationalen Varietät zugeschrieben sind, überprüft, ob diese Zuschreibung korrekt ist, d.h. sie sich wirklich auf den Schweizer Raum beschränken oder aber in (bestimmten Regionen von) Österreich und Deutschland ebenfalls bekannt sind. Dabei werden grenzüberschreitend besonders der alemannische und der bairische Raum berücksichtigt, um zu untersuchen, wie groß jeweils arealer und nationaler Einfluss sind.

\section{Helvetismus und phraseologischer Helvetismus}

Nach der Betrachtung der Aufteilung der deutschen Sprache in verschiedene räumliche Varietäten soll der Blick auf die Bezeichnung der sprachlichen Einheiten oder Merkmale ${ }^{5}$, die diesen Regionen zuzuordnen sind, d.h. die nationalen Varianten, gewendet werden. Auf nationaler Ebene bezeichnen Helvetismen sprachliche Varianten, die für die Standardsprache der Deutschschweiz charakteristisch sind, Austriazismen stehen für österreichische und Teutonismen für bundesdeutsche Besonderheiten. Sprachliche Einheiten, die nicht auf eine oder zwei nationale Varietäten begrenzt sind, werden in diesem Artikel als gemeindeutsch bezeichnet. ${ }^{6}$ Was aber heißt 
„für die Standardvarietät der Deutschschweiz charakteristisch“? Darin stecken zwei Komponenten:

1. Die Zugehörigkeit zur Standardsprache. Nach Burger (1995: 13) sind (phraseologische) Helvetismen ,standardsprachlich akzeptierte lexikalische und phraseologische Besonderheiten der Schweiz". Ammon (1986: 17-39) diskutiert im Hinblick auf den standardsprachlichen Aspekt eine Reihe potentieller Definitionsmerkmale, nämlich Überregionalität, Verwendung durch die Oberschicht, Invarianz, Ausbau, Schriftlichkeit, und hebt als zentrales Merkmal die Kodifizierung der sprachlichen Einheiten hervor. Neben dieser betont Koller (1999: 156) besonders das Bewusstsein der Sprechenden um ihre nationale Varietät als konstituierendes Kriterium für diese Varietät. ${ }^{7}$ Diese beiden Merkmale werden in den Kapiteln 3 und 5.2 beziehungsweise 4 und 5.3 in diesem Artikel ausführlich behandelt.

2. Die geografische Verbreitung der sprachlichen Einheiten oder Merkmale. Diese wird sowohl durch eine maximale als auch eine minimale Erstreckung definiert. Haas (1982: 114) zielt auf die maximale Erstreckung, wenn er lexikalische Helvetismen als ,,jene Wörter, die ausschließlich in der Schweiz vorkommen" charakterisiert, Ammon (1986: 17-22) spricht eine Mindestgrenze an, indem er innerhalb der Schweiz eine überregionale geografische Verbreitung verlangt (zur geografischen Verbreitung exemplarischer Phraseme siehe Kapitel 2).

Auf der arealen Ebene sind für diese Arbeit die Begriffe Alemannismus (zum alemannischen Dialektraum gehören: die Deutschschweiz, Liechtenstein, das österreichische Bundesland Vorarlberg, die südlichen zwei Drittel Baden-Württembergs und das südwestliche Bayern, besonders Schwaben, siehe Ammon 1995) und Bavarismus (aus Österreich, ohne Vorarlberg, sowie Bayern, besonders Ober- und Niederbayern, Oberpfalz, Oberfranken, siehe Ammon 1995) von Bedeutung.

Bei einem phraseologischen Helvetismus handelt es sich um ein standardsprachliches Phrasem der deutschen Schweiz. ${ }^{8}$ Phraseme und ihr Vorkommen in nationalsprachlichen Varietäten wurden empirisch bisher sehr zurückhaltend untersucht (und noch weniger in arealen Varietäten). Zu phraseologischen Helvetismen siehe Arbeiten von Burger (1995, 1996, 1998) und Häcki Buhofer (1998), zu Austriazismen von Földes (1992, 1996), zu Teutonismen von Schmidlin (2004, 2007).

Bei der Arbeit mit phraseologischen Helvetismen ergeben sich zwei zentrale Probleme, die bereits Burger (2007: 209f) anspricht: Einerseits gibt es in der 
Schweiz wie in Österreich Phraseme, die als nationale Varianten klassifiziert werden, sich aber über die Nationengrenzen hinaus (besonders den alemannischen und bairischen Dialektraum) erstrecken (siehe auch Meyer 2006: 20; Földes 1992: 11). ${ }^{9}$ Diese terminologischen Ungenauigkeiten hinsichtlich der Verbreitung der Phraseme sind auf bisher fehlende gesicherte empirische Daten zurückzuführen. Andererseits bereitet die Abgrenzung standardsprachlicher von aus der Mundart übersetzten Phrasemen Schwierigkeiten (Burger 1998: 50, 2007: 218), weil die Informanten in der Abgrenzung häufig unsicher sind und die Wörterbücher in Folge ebenfalls Schwierigkeiten bei der Zuordnung haben. Dieses Abgrenzungsproblem konnte bisher nicht gelöst werden ${ }^{10}$ und wurde auch in der im Folgenden beschriebenen empirischen Untersuchung, in der ausschließlich mit Material gearbeitet wurde, das in Wörterbüchern als standardsprachlich klassifiziert war, wiederholt thematisiert. ${ }^{11}$

\section{Die Kodifizierung nationaler Varianten}

Als zentrale Bedingung für die Zugehörigkeit einer sprachlichen Einheit oder eines Merkmals zur Standardsprache und damit als Voraussetzung für eine Klassifizierung als nationale Variante wurde in Kapitel 2 die Kodifizierung angesprochen. Ammon diskutiert bereits 1986 (37-50) ausführlich Bedeutung und Relevanz einer Kodifizierung der standardsprachlichen Einheiten. Kodifizierung definiert er (1986: 43) als „,die Darstellung der Sprachnormen [...] in Regeln“, die präskriptiven Charakter haben. Im Jahr 1995 (250-253) nennt derselbe Autor explizit, aber nicht abschließend, diejenigen Wörterbücher, in die ein Helvetismus aufgenommen und in denen er als solcher klassifiziert sein muss, um als kodifiziert zu gelten. Dazu gehören in erster Linie eine Reihe von Duden-Bänden (Dudenredaktion 1990, 1991; Schweizer Schülerduden 1978, 1980), aber auch verschiedene andere Nachschlagewerke (z.B. Siebs 1969). Für Phraseme werden speziell Dudenredaktion (1992) und Meyer (1989, ebenfalls Duden-Reihe) angeführt. Ist eine Einheit in diesen Wörterbüchern nicht verzeichnet, so kann sie ebenfalls als Helvetismus gelten, wenn sie ,aufgrund einer anderen Quelle [...] als Helvetismus identifizierbar" ist (Ammon 1995: 253). Ammon (ebd.) geht davon aus, dass „Helvetismen [...] außerhalb ihres eigenen nationalen Zentrums wenig bekannt [sind], abgesehen von einzelnen Ausnahmen“. Wenn diese Annahme stimmt, dann sollte eine Befragung nach der Bekanntheit von Phrasemen für tatsächliche Helvetismen außerhalb der Schweiz sehr geringe Werte liefern, die mit einer ebenfalls sehr geringen Verwendungshäufigkeit korrelieren sollten. 
Das Wörterbuch, das diese Kodifizierungen beinhaltet, bezeichnet Ammon (1986: 49) als „linguistisches Regelwerk [...] für mindestens einen grammatischen Rang" (d.h. Schreibung, Aussprache, Grammatik oder Lexik, siehe Fußnote 5). Die Regeln haben offizielle Gültigkeit und werden beispielsweise in der Schule vermittelt. Auf der einen Seite hat die Kodifizierung also präskriptive Verbindlichkeit, auf der anderen Seite sollte sich das Regelwerk möglichst weitgehend an der Sprachwirklichkeit orientieren. ${ }^{12}$ Dieses Ziel spricht Drosdowski im Vorwort des Herausgebers zur Duden-Grammatik (Dudenredaktion 1984: o. S.) für ein linguistisches Regelwerk explizit an: „,[Die Grammatik] beschreibt primär, sie führt die Breite des Üblichen vor, verschweigt nicht konkurrierende Wortformen und Verwendungsweisen, sondern erläutert sie, und sie achtet darauf, daß Sprachgebrauch und kodifizierte Norm nicht auseinanderklaffen."

Es ist also von einer gegenseitigen Einflussnahme von Kodifizierenden und Sprachgemeinschaft auszugehen. Wie dicht Wörterbuch und Sprachgebrauch aber tatsächlich beieinander liegen, hängt wesentlich von den Zielen und Methoden der Lexikografen sowie vom Alter des Wörterbuchs ab.

In diesem Artikel sollen Wörterbuch und sprachliche Wirklichkeit in der lexikografischen Praxis am Beispiel phraseologischer Einheiten untersucht werden. Für die lexikografische Seite stehen diejenigen aktuellen (phraseologischen) Nachschlagewerke, die in Ammons Sinn gegenwärtig die relevanten Wörterbücher für eine Kodifizierung von Helvetismen sind und die als Grundlage für die hier vorgestellte Arbeit dienten: die Neuauflage des Dudenbandes 11 (Dudenredaktion 2008), die Neuauflage von Meyer (2006) sowie das Variantenwörterbuch (Ammon et al. 2004, das erst rund 10 Jahre nach dem oben zitierten Beitrag von Ammon erschien). Es handelt sich bei allen drei Werken um relativ neue Ausgaben oder Überarbeitungen, die unterschiedlich differenziert ihr methodisches Vorgehen referieren. Die sprachliche Wirklichkeit wird anhand von Befragungsdaten in Kapitel 5 vorgestellt.

Prinzipiell gibt es drei Möglichkeiten, wie Phraseme Eingang in Wörterbücher finden können:

1. Sie können aus anderen Wörterbüchern und älteren Auflagen übernommen werden,

2. sie können aufgrund von Expertenwissen (u.a. der Phraseografen) aufgenommen oder

3. aufgrund empirischer Daten, d.h. anhand von Korpusanalysen oder Informantenbefragungen, Eingang ins Wörterbuch finden. 
Ziel aller drei hier genannten Wörterbücher war die Abbildung des aktuellen Sprachgebrauchs (Dudenredaktion 2008: 15; Meyer 2006: 19; implizit Ammon et al. 2004: VII). Methodisch haben die Bearbeiter des Dudenbandes sowohl auf die bereits vorhandenen lexiko- wie phraseografischen Werke, d.h. die vorangehende Auflage des Bandes sowie auf andere Duden-Wörterbücher, und einschlägige Fachliteratur zurückgegriffen als auch wurden empirische Daten, auf der Grundlage korpuslinguistischer Analysen im Duden-Korpus, herangezogen (ebd.). Meyer (2006: 20f) gibt als Quellen einerseits das eigene Expertenwissen sowie eine umfassende Belegsammlung (aus Zeitungen, Zeitschriften, Sachbüchern und (Fach-) Literatur, aus der sich keine Gebrauchshäufigkeiten ablesen ließen) an. Ammon et al. (2004: VIIf) schließlich rekurrieren ebenfalls auf Expertenwissen (nämlich sowohl der 14 Autor(inn)en als auch einer Reihe weiterer Mitarbeitender aus unterschiedlichen Teilen des deutschsprachigen Raums) sowie auf Internetrecherchen. Weitere Publikationen der einzelnen Autoren und Autorinnen liefern spezifischere Informationen zum Vorgehen: Das Expertenwissen wurde genutzt, indem umfangreiches schriftsprachliches nicht-digitales Quellenmaterial von den einzelnen Bearbeitern aus der Fremdperspektive exzerpiert wurde. D.h. beurteilt wurde je eine fremde Varietät (Bickel 2006; Schmidlin 2004a). Außerdem wurden die Zuweisungen der Wörter und Phrasen zu den jeweiligen Varietäten anhand der Vorkommensfrequenzen der Varianten im Internet in den jeweiligen Domänen in AltaVista und Google rücküberprüft (Bickel 2006; Hofer 2003).

Umfassende Informantenbefragungen, die etwas über die kleinräumigere geografische Verbreitung oder das Bewusstsein der Sprechenden um die nationale Varietät aussagen könnten, wurden, soweit ersichtlich, in keinem der Wörterbuchprojekte durchgeführt. Die beste Chance auf eine in groben Zügen zuverlässige räumliche Zuordnung hat das Variantenwörterbuch, das mit einer räumlich gut gestreuten und relativ großen Zahl von Experten arbeitet. Die Analyse der Vorkommenshäufigkeit der Varianten, die mit Hilfe der Suchmaschinen ermittelt wurden, erlaubt ebenfalls nur eine grobe Zuordnung auf der Ebene der nationalen Varietäten, aber keine Differenzierung innerhalb dieser. So kann beispielsweise die Bekanntheit eines Phrasems im grenznahen Raum (z.B. im alemannischen Teil Deutschlands) nicht zufriedenstellend berücksichtigt werden, da die Frequenzangabe sich auf den gesamten bundesdeutschen Raum bezieht und die Konzentration in einem Teilgebiet nicht sichtbar macht. Zum Bewusstsein der Sprechenden hinsichtlich ihrer Varietät beziehungsweise der gängigen Varianten kann mit den verwendeten Verfahren ebenfalls keine Aussage gemacht werden. 
Die erste Frage, die in Kapitel 5 anhand von Daten aus einer Informantenbefragung exemplarisch beantwortet werden soll, bezieht sich auf die räumliche Verbreitung einer Reihe phraseologischer Helvetismen innerhalb wie außerhalb der Schweiz und damit darauf, wie genau die o.g. Wörterbücher die sprachliche Wirklichkeit wiedergeben. Wir wollen uns also nicht wie Ammon damit zufrieden geben, dass die Phraseme in Wörterbüchern kodifiziert sind, sondern möchten die sprachliche Wirklichkeit direkt und eben nicht über den Umweg der Wörterbücher untersuchen, um herauszufinden, ob die als Helvetismus kodifizierten Varianten tatsächlich nationale oder aber eventuell auch grenzüberschreitende areale Einheiten sind.

\section{Das Bewusstsein der Sprechenden}

In Kapitel 2 wurde neben der Kodifizierung mit dem Bewusstsein der Sprechenden um ihre nationale Varietät bereits ein weiteres Kriterium für die $\mathrm{Zu}$ gehörigkeit einer sprachlichen Variante zur Standardsprache und damit als Bedingung für die Klassifizierung als Helvetismus genannt (siehe Koller 1999: 156). In diesem Bereich lassen sich zwei Ebenen unterscheiden: einerseits ein prinzipielles Bewusstsein auf der Ebene der nationalen Varietät, wie es Koller anspricht, und andererseits ein konkretes Bewusstsein auf der Ebene der sprachlichen Einheiten, in unserem Falle der phraseologischer Helvetismen.

Auf der Ebene des Varietätenbewusstseins spricht Koller (1999: 156) dem Schweizer Standarddeutschen den Status einer nationalen Varietät $\mathrm{ab}$, da sie den Deutschschweizern nicht hinreichend bewusst sei. Zudem finde keinerlei Identifikation der Sprechenden mit der Schweizer Schriftsprache, sondern vielmehr mit den Dialekten (1999: 139), d.h. mit regionalen Räumen anstatt mit einem nationalen (Teil-)Raum statt. Das Plurizentrizitätsbewusstsein der Deutschschweizer in einer empirischen Studie erfragt und getestet (Lesen und Beurteilen von Testsätzen mit Helvetismen auf ihre Korrektheit) hat Scharloth (2004, 2005). Während in einer Befragung 99,7\% seiner Informanten angaben, dass sich Schweizer und Deutsche beim Sprechen der Standardsprache unterscheiden, kam Scharloth auf der Grundlage von Sprachbewusstseinstests zu dem Ergebnis, dass Schweizer Helvetismen systematisch (in gut 70\% der Fälle) als schlechtere oder nicht korrekte Standardsprache und eben nicht als gleichberechtigte Schweizer Varianten einstuften (2004: o. S.). Scharloth schließt aus diesen Ergebnissen, dass von einem Plurizentrizitäts- oder 
Varietätenbewusstsein der Deutschschweizer nicht gesprochen werden kann (2004: o. S., 2005: 263).

Auf der Ebene des Helvetismenbewusstseins geht wiederum Koller (1999: 159) davon aus, dass die Schweizer Varianten den Sprechenden sehr unterschiedlich bewusst sind. Zum Teil seien sie es gar nicht bewusst, zum Teil würden sie in der Schule wie Vokabeln erlernt und zum Teil würden sie auch als dialektal statt als standardsprachlich eingestuft. Im Bereich der Phraseologie geht Burger (1998: 52) davon aus, dass phraseologische (standardsprachliche) Helvetismen (im Gegensatz zu den aus dem Dialekt in die Standardsprache übersetzten Phrasemen) den Sprechenden in der Regel nicht bewusst sind. Eine Befragung von 79 Schweizer Germanistikstudierenden, die 15 gemeindeutsche und 15 Schweizerische Phraseme mit hohem Bekanntheitsgrad hinsichtlich ihrer nationalen Verbreitung zuordnen sollten, ergab, dass es sich bei den am schlechtesten zugeordneten fünf Phrasemen um Helvetismen handelte ( 23 bis 57\% richtige Zuordnung), d.h. dieses Drittel der allgemein bekannten Schweizer Phraseme lag sehr deutlich unter oder nur gering über der zufälligen Trefferwahrscheinlichkeit von 50\%. Der Mittelwert der korrekten Einstufung der 15 phraseologischen Helvetismen lag bei $76 \%$. Hinsichtlich einer Verallgemeinerbarkeit dieses Ergebnisses von Philologiestudierenden geht Burger von einem allgemein eher bescheidenen Bewusstsein für Helvetismen in der gesamten Sprachgemeinschaft aus (1998: 72-74).

Was das Verhältnis dieser beiden Bewusstseinsebenen zueinander angeht, sollte man davon ausgehen können, dass sie prinzipiell korrelieren. Je größer das Bewusstsein auf der Variantenebene (d.h. je mehr Varianten der Sprechende als nationale Besonderheit auf der Ebene der Standardsprache einstuft), desto größer sollte auch das Varietätenbewusstsein sein (d.h. desto größer die Wahrscheinlichkeit, dass er/sie ein plurizentrisches Bewusstsein entwickelt hat). Und auf der anderen Seite sollte ein Informant mit ausgeprägtem Varietätenbewusstsein eher dazu neigen, Varianten als national einzustufen.

Da in der im Folgenden beschriebenen empirischen Untersuchung nur das Helvetismenbewusstsein, nicht aber das Varietätenbewusstsein erfragt wurde, ist letzteres nur tendenziell im Rückschluss von der Varianten- auf die Varietätenebene möglich. Die zweite Frage an die empirischen Daten bezieht sich also auf das Sprachbewusstsein auf Phrasemebene, d.h. wie bewusst ist es den Schweizer Sprechenden, dass es sich bei den abgefragten Phrasemen um Helvetismen handelt? Dieser Frage wird in Abschnitt 5.3 nachgegangen. 


\section{Empirische Daten zur räumlichen Verbreitung phraseologischer Helvetismen und zum Bewusstsein der Sprechenden}

\subsection{Material und Methoden}

$\mathrm{Da}$ in dieser Studie eine möglichst genaue räumliche Zuordnung der Informanten (zu Kantonen oder Bundesländern) und damit ihrer Angaben vorgenommen werden sollte, wurde mit einer Informantenbefragung gearbeitet. Die Daten wurden in einer Online-Befragung ${ }^{13}$ zur Bekanntheit ${ }^{14}$ einer Auswahl von phraseologischen Helvetismen im Frühling 2009 gemeinsam mit Germanistikstudierenden der Universität Basel erhoben. ${ }^{15}$ Die vorgestellten Phraseme wurden mit den Studierenden als typische phraseologische Helvetismen ausgewählt (Expertenwissen) und sind in mindestens einem der in Kapitel 3 genannten Wörterbücher (Ammon et al. 2004; Dudenredaktion 2008; Meyer 2006) als Schweizer Varianten kodifiziert. Mit dem Fragebogen wurde im Multiple-choice-Verfahren die Form (in Ergänzungstests) ${ }^{16}$ beziehungsweise die Bedeutung ${ }^{17}$ von 18 helvetischen Phrasemen erhoben (Auflistung siehe Anhang 1). Außerdem wurden die Informanten zu einem Teil der Phraseme gefragt, ob es sich ihrer Meinung nach um Helvetismen handle, ${ }^{18}$ und sie wurden gebeten, eine Reihe von soziodemografischen Angaben zu machen (für eine areale Zuordnung: Sozialisierung mit/ohne Dialekt und Ort, an dem der größte Teil des Lebens verbracht wurde, sowie Alter, Geschlecht, Ausbildung). Zur Teilnahme an der Befragung wurde in einer kostenlosen Online-Zeitung aufgerufen und die Fragebögen wurden im Schneeballprinzip als E-Mails an Bekannte verschickt.

Im folgenden Kapitel werden exemplarisch die Ergebnisse zur räumlichen Verbreitung von sechs der abgefragten Helvetismen vorgestellt (Auflistung der Phraseme siehe Tabelle 1), die teilweise Varianten zu bundesdeutschen Phrasemen und teilweise eigenständige Schweizer Phraseme ohne strukturell ähnliches Äquivalent im Bundesdeutschen darstellen (zu diesen Kategorien siehe Burger 1995: 17). Diese sechs Phraseme wurden wegen ihrer unterschiedlichen räumlichen Verbreitung ausgewählt.

An der Befragung haben sich rund 1000 Personen beteiligt, von denen 981 bei der Auswertung berücksichtigt werden konnten. ${ }^{19} 863$ von ihnen stammten aus der Schweiz (bis auf Obwalden wurden alle Deutschschweizer Kantone abgedeckt), 118 aus Deutschland, Österreich und Liechtenstein (zur Verteilung der Informanten auf die einzelnen Kantone und Bundesländer siehe Anhang 2). Wegen der geringen Teilnehmerzahlen aus Österreich in dieser Untersuchung (5) ist eine gewisse Vorsicht bei der Interpretation der österreichischen Daten geboten. 
Helvetismen: Nationale und areale Varianten?

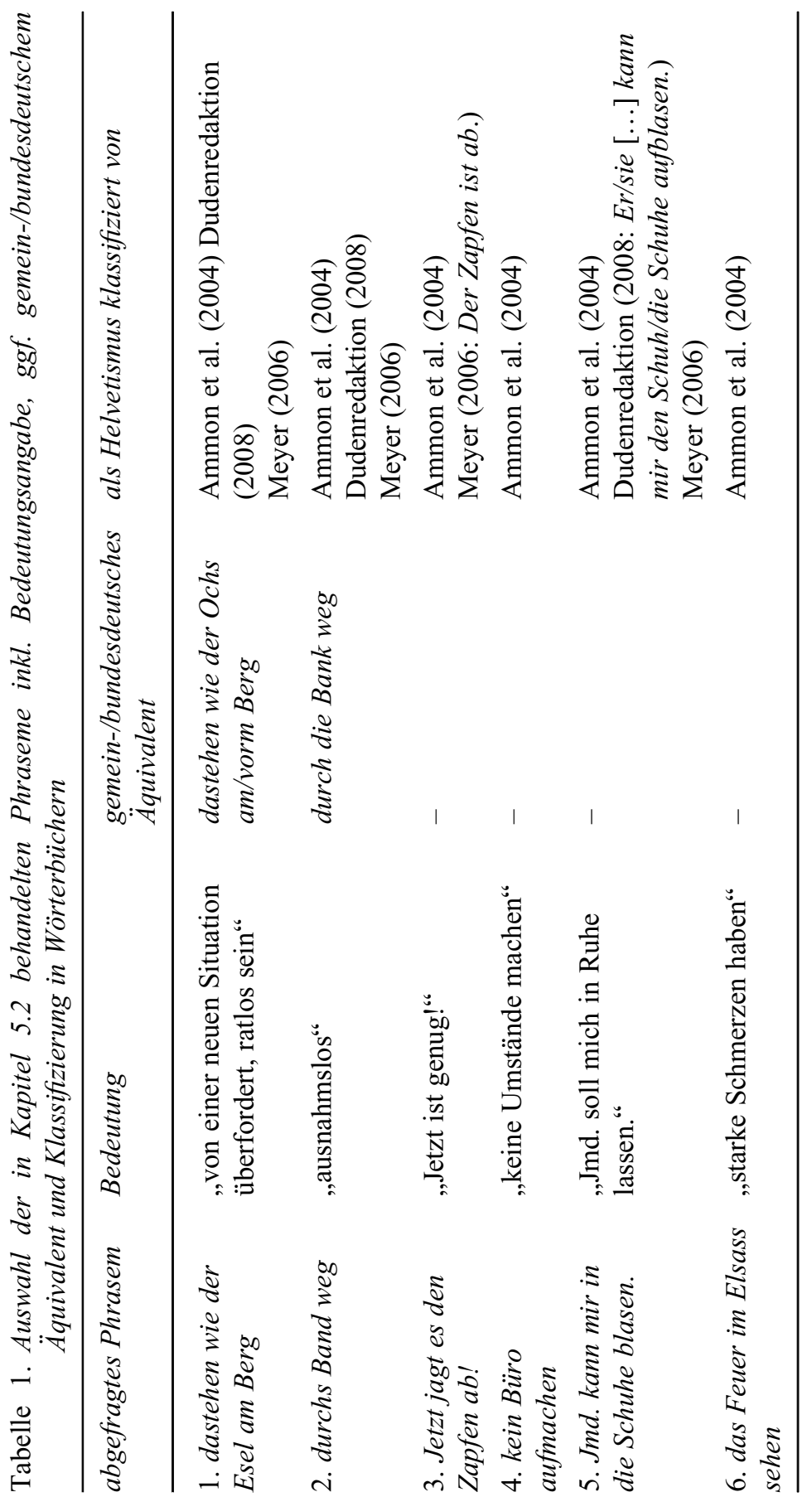




\subsection{Die räumliche Verbreitung der phraseologischen Helvetismen}

Ein erster Blick auf den Bekanntheitsgrad der sechs exemplarischen Phraseme innerhalb und außerhalb der Schweiz (siehe Abbildung 2) zeigt, dass alle Phraseme in der Schweiz deutlich mehr Informanten bekannt waren als in Österreich und Deutschland. Dieser Unterschied lässt sich mit Hilfe eines t-Tests auch statistisch nachweisen $(p=0.007)$. Diese Verteilung spricht prinzipiell für eine Kategorisierung der Phraseme als Helvetismen. Der Bekanntheitsgrad der einzelnen Phraseme außerhalb der Schweiz fällt allerdings sehr unterschiedlich aus. Was besonders bei den Phrasemen 4 und 5 einen ersten Hinweis darauf gibt, dass die Bezeichnung „Helvetismus“ unter Umständen zu eng gefasst sein könnte.

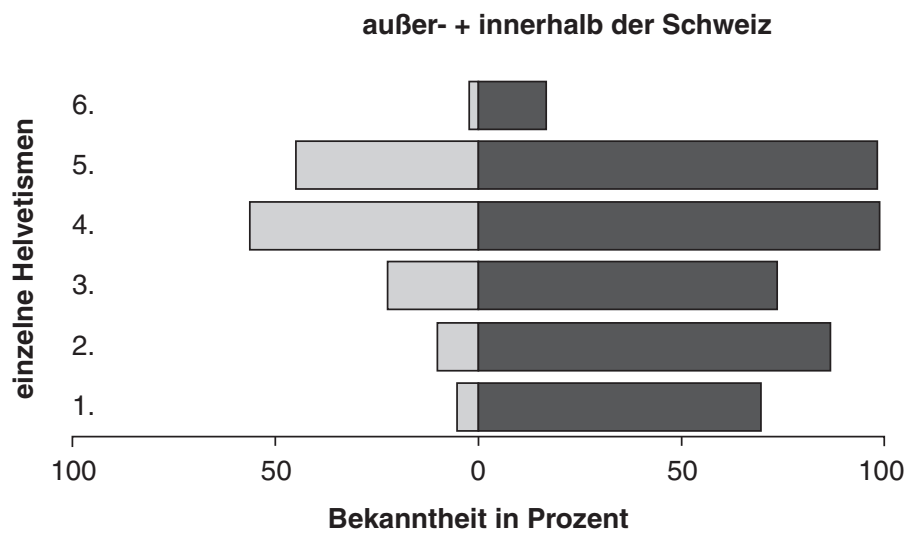

Abbildung 2. Bekanntheit der sechs exemplarischen Phraseme außerhalb (graue Balken links, durchschnittlicher Bekanntheitsgrad 23\%) und innerhalb (schwarze Balken rechts, durchschnittlicher Bekanntheitsgrad 74\%) der Schweiz (die Nummerierung entspricht derjenigen in Tabelle 1 und der folgenden Beispiele).

Zu den folgenden Karten ${ }^{20}$ (Abbildungen 3-8)

$\mathrm{Da}$ in dieser Untersuchung zwei Ebenen der räumlichen Verbreitung untersucht werden sollten, nämlich sowohl die Verteilung innerhalb der Nationen, insbesondere in der Schweiz, als auch in den Arealen des Alemannischen und des Bairischen, müssen aus den kartografischen Darstellungen beide Bereiche ersichtlich sein. Es werden daher in den Abbildungen 3-8 die Schweizer Informanten nach Kantonen gezeigt, die deutschen Informanten 
den drei Bereichen Baden-Württemberg ${ }^{21}$, Bayern ${ }^{22}$ oder Rest-Deutschland zugeordnet. Da aus Österreich und Liechtenstein mit fünf und sieben nur sehr wenige Informanten teilgenommen haben, werden diese nach Ländern zusammengefasst. ${ }^{23}$

Beispiel 1: dastehen wie der Esel am Berg ${ }^{24}$

Dieses Verbalphrasem mit der Bedeutung „von einer neuen Situation überfordert, ratlos sein“, das eine Variante zu dastehen wie der Ochs am/vorm Berg darstellt, wird sowohl von Ammon (2004) als auch von der Dudenredaktion (2008) und Meyer (2006) als Helvetismus klassifiziert (siehe Tabelle 1). Die empirischen Daten (kartografisch dargestellt in Abbildung 3) zeigen in der Deutschschweiz einen Bekanntheitsgrad zwischen 20 und 100\%, wobei abgesehen von den Kantonen Bern, Freiburg und im Wallis im Westen und im Thurgau und in Graubünden im Osten, wo die Bekanntheit zwischen 20 und 60\% liegt, ein Bekanntheitsgrad zwischen 60 und 100\% erreicht wird. In den Gebieten außerhalb der Schweiz, d.h. sowohl in Österreich (und Liechtenstein) als auch im gesamten Raum der Bundesrepublik Deutschland, also auch im Alemannischen und Bairischen, ist dieses Phrasem nicht bekannt (Bekanntheit $<20 \%$ ). ${ }^{25}$

Auf der Grundlage dieser überregionalen Verbreitung in der Schweiz und der (tendenziellen) Unbekanntheit außerhalb der Schweiz ist dastehen wie der Esel am Berg, wie in den Wörterbüchern geschehen, eindeutig als (absoluter ${ }^{26}$ Helvetismus einzustufen.

\section{Beispiel 2: durchs Band weg}

Dieses Phrasem in der Bedeutung ,ausnahmslos“ wird im bundesdeutschen Raum in der Variante durch die Bank weg verwendet. Durchs Band weg wird von allen drei konsultierten Wörterbüchern als Helvetismus eingestuft. Aus den Informantendaten (siehe Abbildung 4.) ergibt sich für die Schweiz ein sehr einheitliches Bild: In der überwiegenden Zahl der Kantone hat das Phrasem einen Bekanntheitsgrad von $81-100 \%$, nur im Wallis und in Nidwalden (auch in Liechtenstein) ist es nur $61-80 \%$ der Befragten bekannt. Jenseits der Schweizer Landesgrenzen ist es in Österreich der Hälfte der Informanten bekannt (41-60\%), im bundesdeutschen Raum hingegen herrscht eindeutig die Variante durch die Bank weg vor (das Schweizer Phrasem wird von nur $0-20 \%$ angegeben).

Da das Phrasem neben der flächendeckenden Verbreitung in der Schweiz auch in Österreich etwa der Hälfte der Informanten bekannt ist, während es in Deutschland nicht vorzukommen scheint, scheint es - unter 


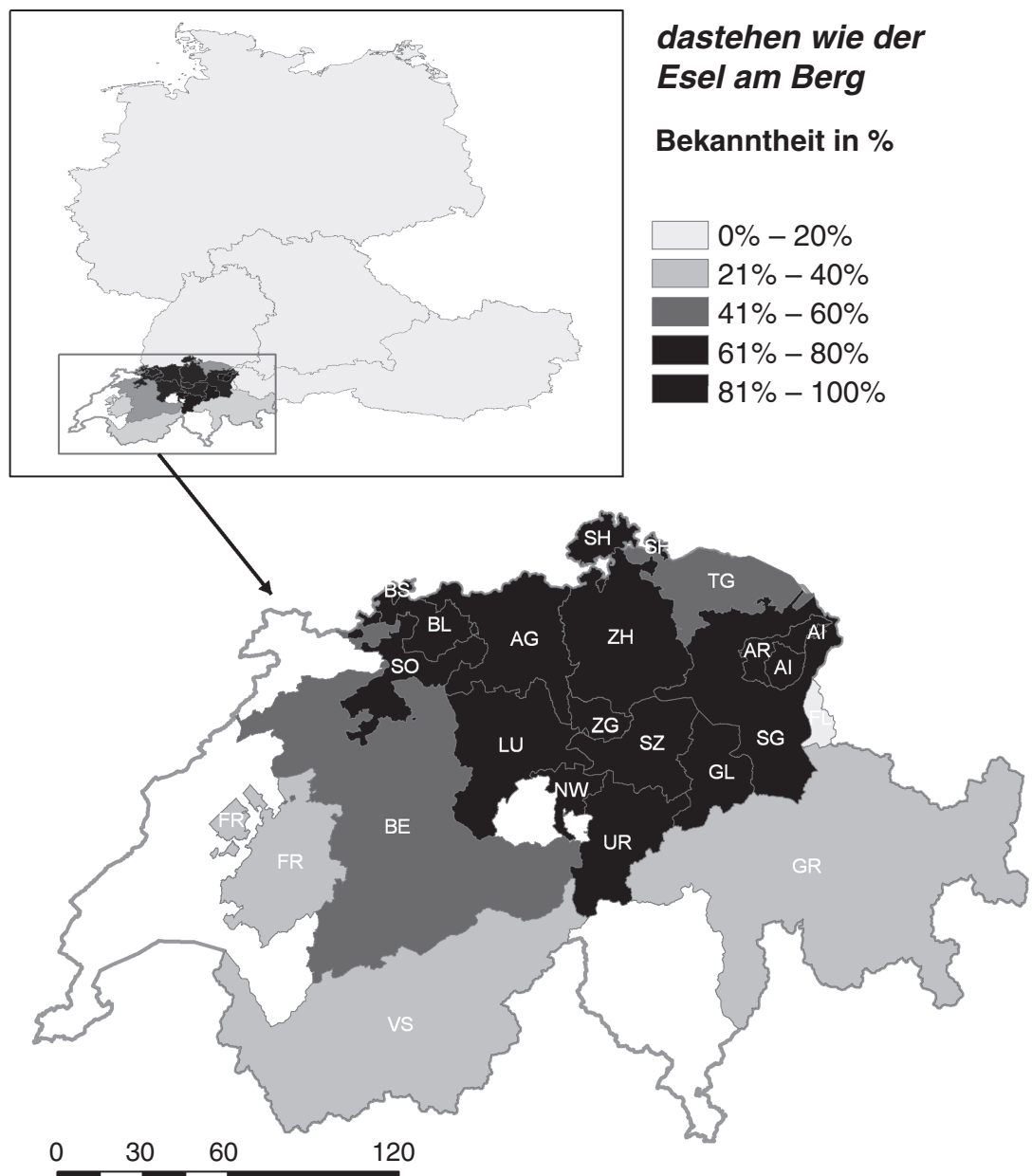

Kilometer

Abbildung 3. Bekanntheit von dastehen wie der Esel am Berg innerhalb und außerhalb der Schweiz

Annahme der Verallgemeinerbarkeit der Angaben der wenigen Informanten auf die Sprachgemeinschaft - korrekter, es statt als Helvetismus als Austro-Helvetismus zu kategorisieren.

Beispiel 3: Jetzt jagt es den Zapfen ab.

Dieses satzwertige Phrasem mit der Bedeutung „Jetzt reicht es!“, ohne strukturell ähnliche gemeindeutsche Variante, wird von Ammon et al. 


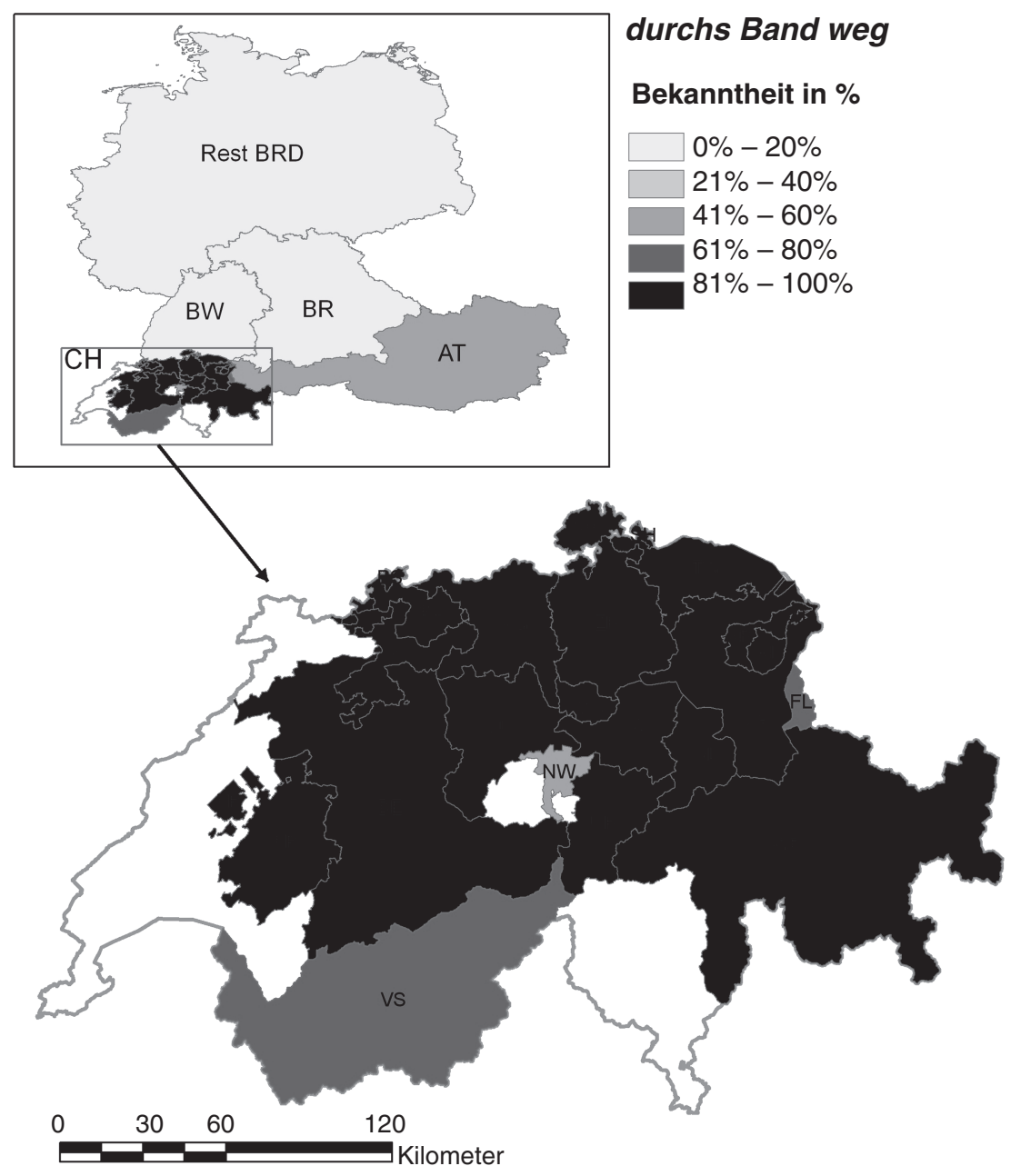

Abbildung 4. Bekanntheit von durchs Band weg innerhalb und außerhalb der Schweiz

(2004) und Meyer (2006, in der Variante: Der Zapfen ist ab.) als Helvetismus klassifiziert, in Dudenredaktion (2008) ist es nicht verzeichnet. Die Visualisierung der Befragungsdaten in Abbildung 5 zeigt für die Schweiz ein uneinheitlicheres Bild als für die vorangehenden Phraseme. In den (auch) deutschsprachigen Westschweizer Kantonen Fribourg, Bern, Solothurn und Wallis sowie in Schwyz und Appenzell Ausserrhoden (wie auch in Liechtenstein) ist es zwischen 81 und 100\% der Befragten bekannt, in den 


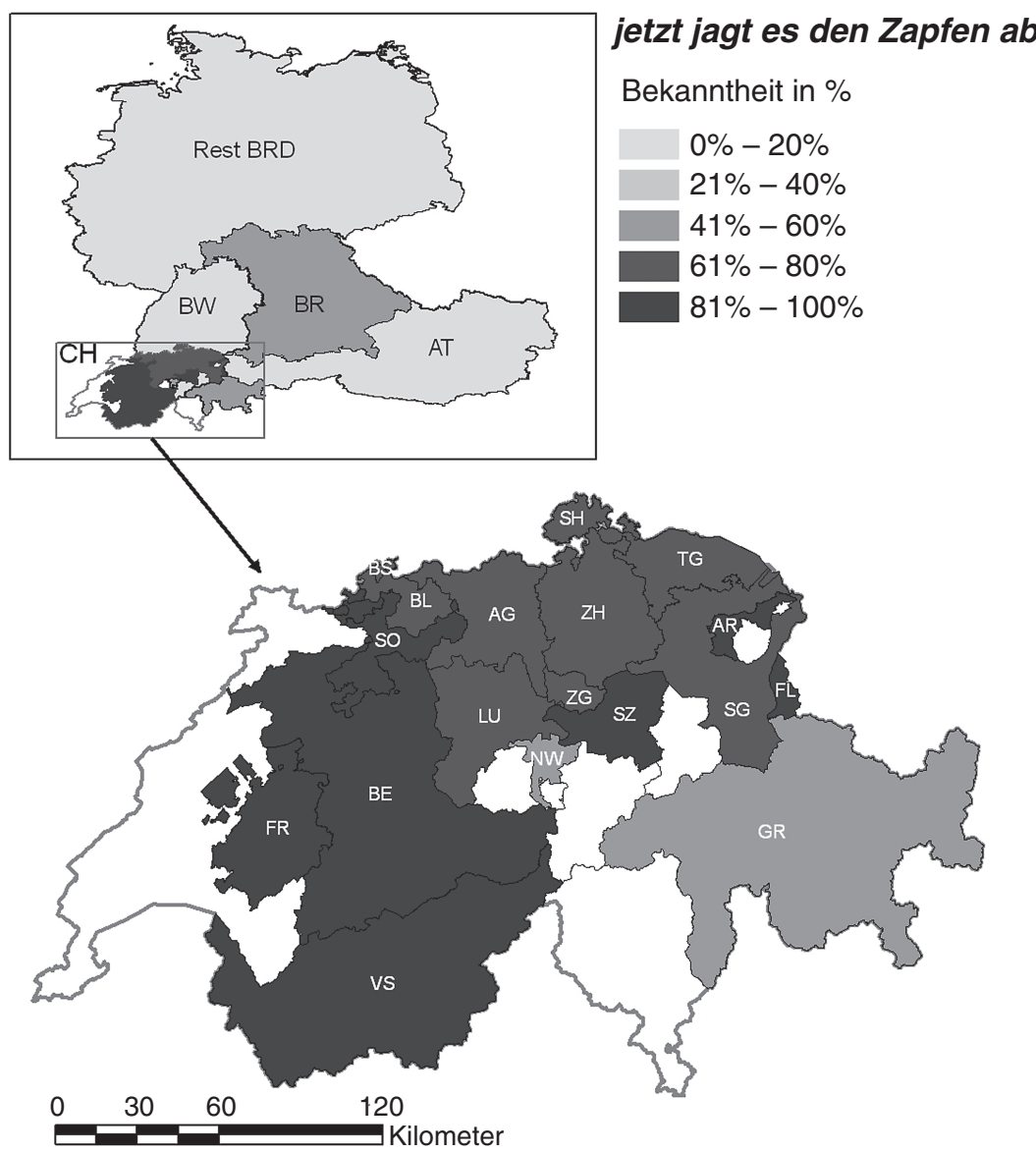

Abbildung 5. Bekanntheit von Jetzt jagt es den Zapfen ab. innerhalb und außerhalb der Schweiz

nördlichen Kantonen (Baselland, Baselstadt, Aargau, Luzern, Schaffhausen, Zürich, Zug, Thurgau, St. Gallen) zwischen 61 und 80\%, in Graubünden zwischen 41 und $60 \%$ und in den übrigen Kantonen $40 \%$ und weniger. Außerhalb der Schweiz kannten nur 41-60\% der bairischen Informanten dieses Phrasem, in den übrigen Regionen war es (nahezu) unbekannt (0-20\%).

Aufgrund der überregionalen Verbreitung in der Schweiz und einem Bekanntheitsgrad von $50 \%$ in Bayern neben der Unbekanntheit im übrigen deutschsprachigen Raum, wird - in Abweichung von den Wörterbüchern vorgeschlagen, dieses Phrasem als Bavaro-Helvetismus zu klassifizieren. 


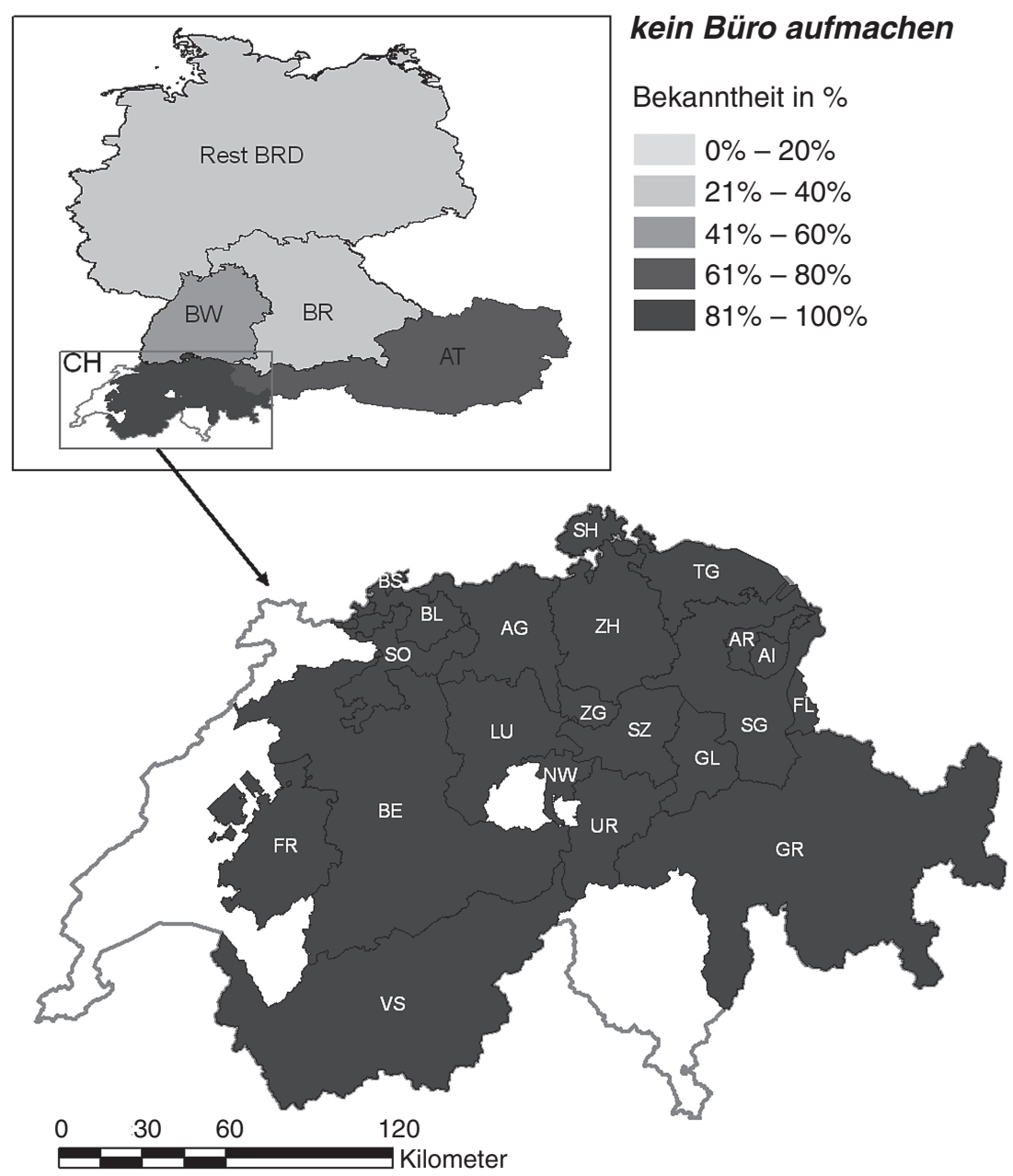

Abbildung 6. Bekanntheit von kein Büro aufmachen innerhalb und außerhalb der Schweiz

Beispiel 4: kein Büro aufmachen

Das Verbalphrasem kein Büro aufmachen mit der Bedeutung „keine Umstände machen", das ebenfalls keine strukturell äquivalente Variante im Gemeindeutschen hat, wird nur von Ammon et al. (2004) als Helvetismus aufgeführt, in den beiden anderen Wörterbüchern fehlt es. Den empirischen Daten zufolge (siehe Abbildung 6) ist das Phrasem in der gesamten Schweiz 
und Liechtenstein allgemein bekannt (81-100\%). Darüber hinaus ist es in Österreich 61-80\% und in Baden-Württemberg 41-60\% der Informanten bekannt. Im übrigen deutschen Raum erreichte es einen Bekanntheitsgrad von $21-40 \%$. Wegen seiner Verbreitung in der ganzen Schweiz und des größeren Bekanntheitsgrades in Österreich und Baden-Württemberg als im übrigen bundesdeutschen Raum wird in Abweichung von Ammon et al. (2004) vorgeschlagen, dieses Phrasem als Austro-Alemannismus zu bezeichnen.

Beispiel 5: Jmd. kann mir in die Schuhe blasen.

Dieses satzwertige Phrasem mit der Bedeutung „Jmd. soll mich in Ruhe lassen." ist in den Wörterbüchern von Ammon et al. (2004) und Meyer (2006) als Helvetismus angegeben. In Dudenredaktion (2008) wird mit der gleichen Bedeutung die Variante Er/sie [...] kann mir den Schuh/die Schuhe aufblasen. (ohne Angabe der räumlichen Erstreckung) genannt. Die Befragung (siehe Abbildung 7) ergab eine allgemeine Bekanntheit dieses Phrasems in der Schweiz wie in Österreich (und Liechtenstein, überall 81-100\%). In Baden-Württemberg und Bayern erreichte es eine Bekanntheit von immerhin $61-80 \%$, im restlichen bundesdeutschen Raum blieb es mit $21-40 \%$ deutlich dahinter zurück.

Aufgrund des hohen Bekanntheitsgrades nicht nur in der Schweiz, sondern in Österreich und auch den beiden südlichen Bundesländern Deutschlands wird vorgeschlagen, statt von einem Helvetismus von einem Phrasem, das im gesamten süddeutschen Raum verbreitet ist, zu sprechen.

\section{Beispiel 6: das Feuer im Elsass sehen}

Als letztes Beispiel sei das Verbalphrasem das Feuer im Elsass sehen, wiederum ohne gemeindeutsche Variante (,große Schmerzen haben“), angeführt. Dieses ist nur bei Ammon et al. (2004) angeführt und als Helvetismus eingestuft. Den Informantenangaben zufolge (siehe Abbildung 8) ist dieses Phrasem nur in wenigen Regionen der Schweiz bekannt. ${ }^{27}$ Es wurde wegen seiner räumlichen Nähe zum Elsass nicht ganz überraschend - in den nordwestlichen Kantonen Baselland und Baselstadt, Aargau, im nördlichsten Kanton Schaffhausen sowie im Wallis von weniger als der Hälfte der Informanten (21-40\%) als bekannt angeführt. In den übrigen Schweizer Kantonen sowie im restlichen deutschsprachigen Raum war es (nahezu) unbekannt (in der Schweiz: 0-20\%, in Österreich, Deutschland und Liechtenstein: 0-4\%).

Aufgrund der regionalen Beschränkung auf wenige Kantone und des auch hier vergleichsweise geringen Bekanntheitsgrads wird vorgeschlagen, 


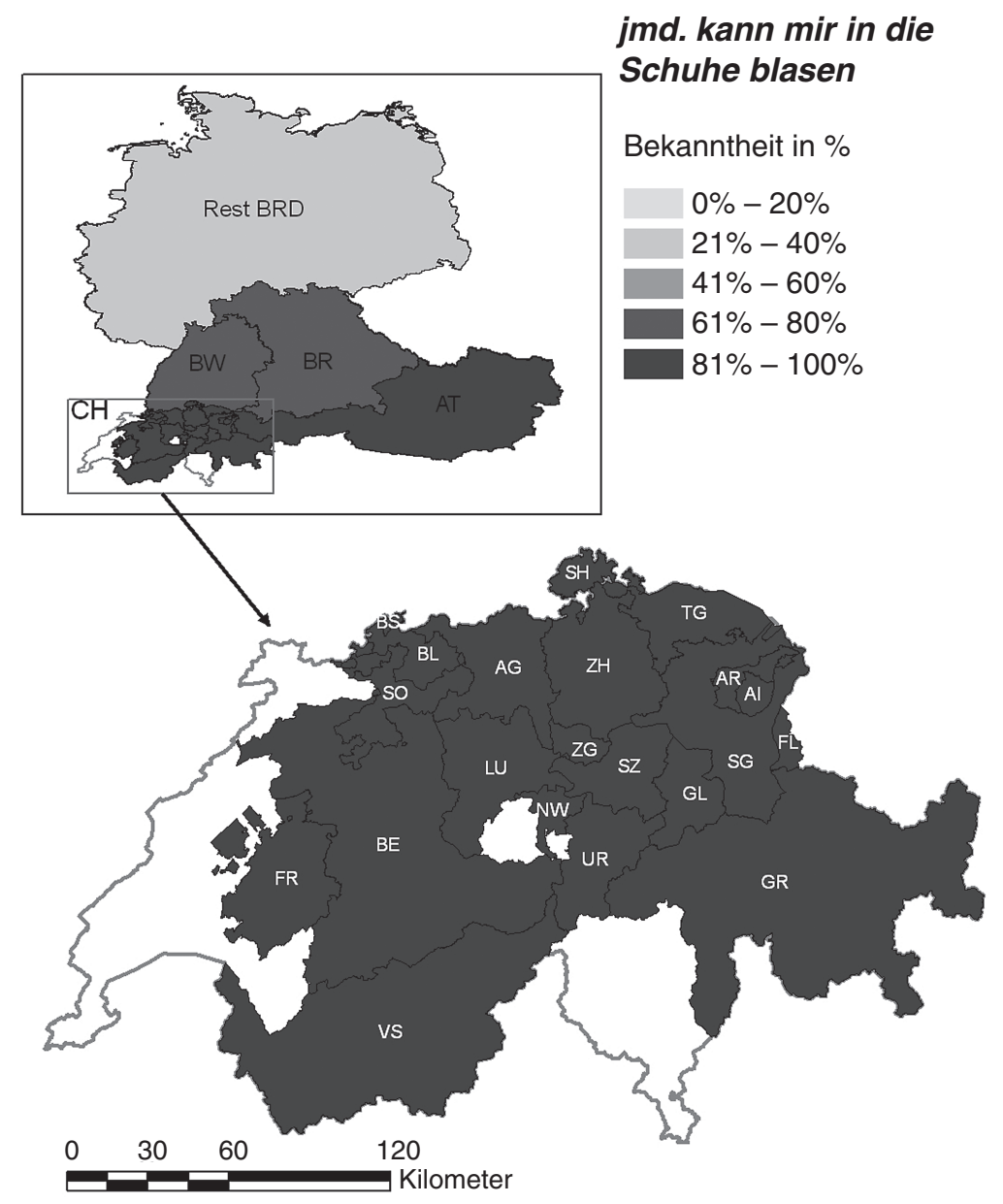

Abbildung 7. Bekanntheit von Jmd. kann mir in die Schuhe blasen. innerhalb und außerhalb der Schweiz

nicht von einem Helvetismus, sondern von einem regionalen Helvetismus oder einem Schweizer Regionalismus zu sprechen. ${ }^{28}$

Ammons (1995: 253) Annahme, dass Helvetismen in der Regel außerhalb der Schweiz wenig bekannt sind, kann - abgesehen von denjenigen Einheiten, die hier als grenzüberschreitend klassifiziert wurden - anhand der phraseologischen Daten bestätigt werden. 


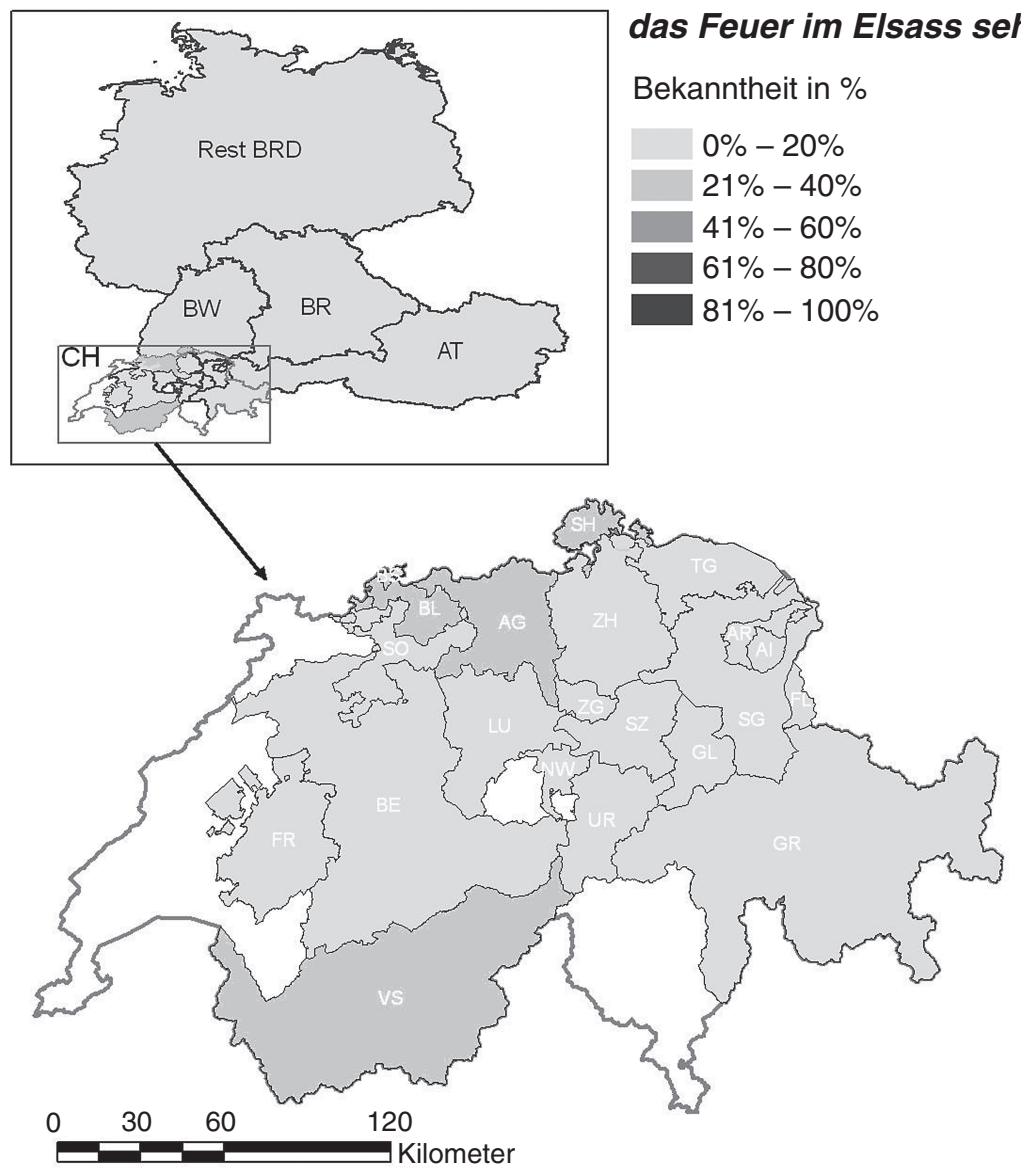

Abbildung 8. Bekanntheit von das Feuer im Elsass sehen innerhalb und außerhalb der Schweiz

\subsection{Das Helvetismenbewusstsein der Sprechenden}

In Kapitel 4 wurde die Frage nach dem Bewusstsein der Sprechenden um den Status der Phraseme als Helvetismus aufgeworfen. Den Ausgangspunkt bildet die Aussage Burgers (1998: 74), der von einem unauffälligen Bestand der Schweizer Varianten und damit einem geringen Bewusstsein für phraseologische Helvetismen ausgeht. Daran schließt sich die Frage an, ob sich Faktoren identifizieren lassen, die mit besonders hohen oder geringen Werten für die Einschätzung eines Phrasems als Helvetismus in Zusammenhang stehen. 


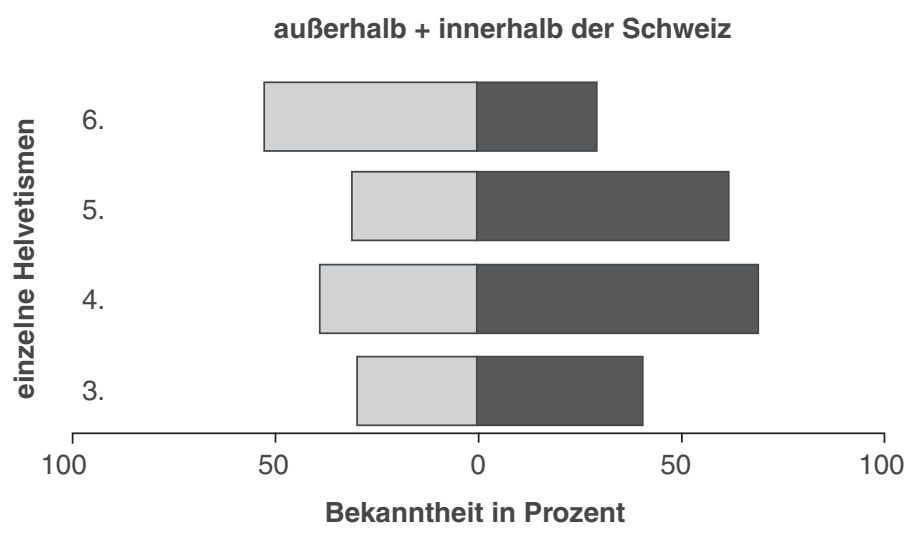

Abbildung 9. Einschätzung von vier der sechs exemplarischen Phraseme als Helvetismus außerhalb (graue Balken links, Mittelwert 49\%) und innerhalb (schwarze Balken rechts, Mittelwert 38\%) der Schweiz. Nr. 3: Jetzt jagt es den Zapfen ab., Nr. 4 kein Büro aufmachen, $N r .5$ Jmd. kann mir in die Schuhe blasen., Nr. 6 Das Feuer im Elsass sehen.

Auf der Datengrundlage von vier der sechs in Tabelle 1 angeführten Phraseme (zu den anderen zwei Phrasemen wurde der Status als Helvetismus nicht abgefragt) wird in Abbildung 9 die Zuordnung als Helvetismus vs. gemeindeutsches Phrasem durch die Informanten dargestellt. Vergleicht man zunächst einmal die varietäteninterne Einstufung der Schweizer Informanten mit der varietätenexternen Beurteilung der deutschen und österreichischen Befragten, lässt sich prinzipiell feststellen, dass diese vier Phraseme (wie auch sieben weitere, hier nicht aufgeführte, die im Fragebogen hinsichtlich der räumlichen Verbreitung einzustufen waren, siehe Fußnote 29) in der Regel in der Schweiz von einem größeren Prozentsatz der Informanten als Helvetismen eingestuft wurden. Das Bewusstsein für Helvetismen scheint also innerhalb der Varietät größer zu sein als außerhalb der Varietät (p-Wert für alle elf Phraseme $\left.{ }^{29}<0.001\right) .{ }^{30}$ Die einzige Ausnahme bildet das Phrasem das Feuer im Elsass sehen.

Die durchschnittliche Klassifizierung der vier Phraseme als Helvetismen beträgt knapp 50\% (und entspricht etwa derjenigen aller 11 Phraseme, die als nationale Varianten zugeordnet wurden: 47\%). Im Vergleich zu den von Burger befragten Studierenden (76\% korrekte Einstufung als Helvetismus, siehe Kapitel 4) liegt der sich aus diesen Daten ergebende Mittelwert wie von Burger für die durchschnittliche Bevölkerung vermutet - deutlich niedriger. 
Im Vergleich dazu wurde ein gemeindeutsches, weniger bekanntes Phrasem (Der Krug geht zum Brunnen, bis er bricht. mit einem Bekanntheitsgrad von $26 \%$ ) von nur $4 \%$ und ein Phantasiephrasem (Der Storch fliegt vorbei.) von 5\% der Schweizer Informanten als Helvetismus eingestuft. Diese geringen Werte könnten ein Hinweis darauf sein, dass die Schweizer Informanten relativ verlässlich ein Phrasem nur dann als Helvetismus einstufen, wenn sie sich bei der Zuordnung sicher sind. Die Werte könnten aber auch mit dem geringen Bekanntheitsgrad dieser beiden Phraseme in Zusammenhang stehen, denn je mehr Informanten eine Einheit kennen, desto mehr können sie begründet als Helvetismus bewerten (ein unbekanntes Phrasem ist schwer zu klassifizieren). Um diese Hypothese zu prüfen, wurden nicht nur die entsprechenden Daten der vier oben genannten, sondern aller elf Helvetismen, die von den Informanten auf ihre räumliche Verbreitung hin beurteilt wurden, korreliert (siehe Abbildung 10). Durch die größere Zahl der Helvetismen konnte die statistische Power erhöht werden. Die Regression belegt deutlich einen statistisch signifikanten linearen Zusammenhang zwischen den Datensätzen $(p=0.008)$ : Phraseme werden mit zunehmendem Bekanntheitsgrad häufigerer korrekt als Helvetismus eingeschätzt (siehe Verlauf der Regressionsgeraden). Das Bestimmtheitsmaß $\mathrm{R}^{2}=0.56$ gibt an, dass immerhin 56\% der Variabilität der Einschätzung als Helvetismus durch den Bekanntheitsgrad der Phraseme erklärt werden.

Diese Korrelation kann ein Grund für die deutlich höheren Bewusstseinswerte in Burgers Studie (1998, siehe Kapitel 4) sein, denn bei den von

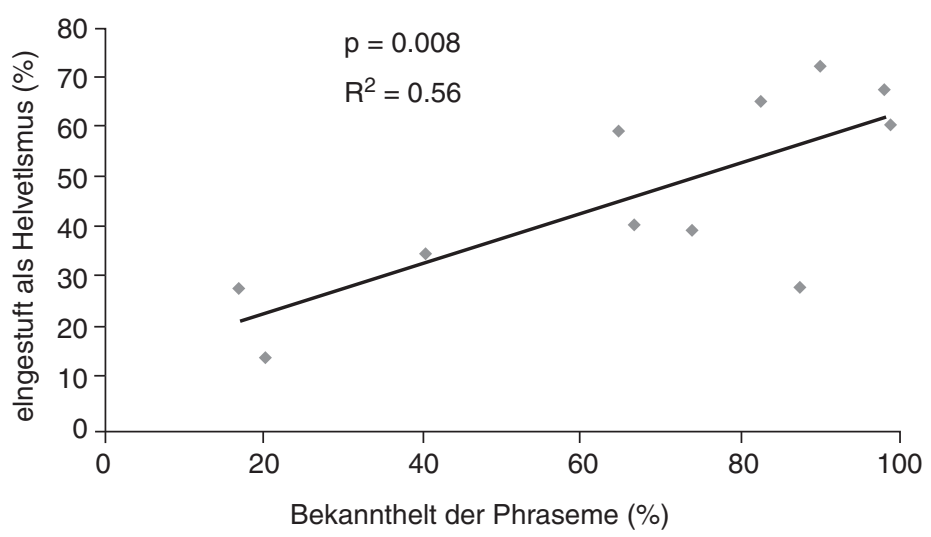

Abbildung 10. Korrelation der Bekanntheit der Phraseme in der Schweiz (in Prozent) und der Einstufung als Helvetismus durch Schweizer Informanten (in Prozent) 
ihm vorgegebenen Phrasemen handelte es sich durchweg um Einheiten mit einem sehr hohen Bekanntheitsgrad (1998: 72). ${ }^{31}$

Dass das Feuer im Elsass sehen in der Schweiz von relativ wenigen Informanten als Helvetismus eingeschätzt wurde, scheint daher auch damit zusammenzuhängen, dass es innerhalb der Schweiz eine geringe räumliche Verbreitung und einen beschränkten Bekanntheitsgrad hat. Der relativ große Anteil Nicht-Schweizer, der dieses Phrasem als Helvetismus ansieht, könnte andererseits in diesem Fall möglicherweise auf die räumliche Nähe der im Phrasem enthaltenen Ortsangabe (Elsass) zur Schweiz zurückzuführen sein, der die Nicht-Schweizer verführt haben könnte, auf seinen Status als Helvetismus rückzuschließen. Solche landestypischen Hinweise (geografische Angaben, aber auch politische, gesellschaftliche u.a. Besonderheiten) können die nationale Zuordnung beeinflussen. Einen ähnlichen Einfluss dürfte es haben, wenn einzelne Komponenten eines Phrasems Helvetismenstatus haben. So ist es beispielsweise bei Jetzt jagt es den Zapfen $a b$. möglich, dass Zapfen als Flaschenverschluss die Informanten zur Klassifizierung des Phrasems als Helvetismus veranlasst hat.

Und schließlich könnte für die Einschätzung auch der Phrasemtyp relevant sein, d.h. ob es sich bei den Phrasemen um eigenständige Schweizer Einheiten (ohne Varianten in den anderen nationalen Varietäten), um Sonderphraseologie (mit Varianten) oder um falsche Freunde handelt (vgl. Fußnote 8). Hier reicht das Material dieser Untersuchung für eine statistische Auswertung nicht aus. Das Bewusstsein im Hinblick auf das einzige im Material enthaltene Phrasem, das zur Sonderphraseologie gehört (in kurzen Hosen dastehen) und das national zugeordnet werden sollte, liegt deutlich unterhalb der in Abbildung 10 enthaltenen Geraden (Bekanntheit $87 \%$, Einstufung als Helvetismus 28\%). Das gilt auch für den phraseologischen falschen Freund jmdm. die Stange halten in der Bedeutung, ,jmdn. unterstützen“ (Bekanntheit 20\%, Einstufung als Helvetismus 14\%). Diese beiden Beispiele weisen im Verhältnis zur Bekanntheit die geringsten Bewusstseinswerte auf, was ein Hinweis darauf sein könnte, dass Schweizer Sonderphraseologie und falsche Freunde den Schweizer Probanden weniger häufig als Helvetismen bewusst sind als die dritte Gruppe der eigenständigen Schweizer Phraseme - was als Hypothese für umfangreichere Untersuchungen dienen kann.

Bisher wurde in diesem Abschnitt vom absoluten, d.h. tatsächlich auf die Schweiz begrenzten Helvetismus ausgegangen. Die in 4.5.2. dargestellten Ergebnisse haben aber gezeigt, dass einige Phraseme durchaus auch in angrenzenden Räumen anderer Nationen geläufig sind. Neben der räumlichen Verbreitung und Bekanntheit innerhalb der Schweiz könnte daher auch ein 
Zusammenhang zwischen der Klassifizierung als Helvetismus und der Verbreitung außerhalb der Schweiz bestehen, denn wenn die Informanten mit einem Phrasem auch grenzübergreifend in Berührung kommen (unter der Annahme, dass Nichtschweizer diese Einheiten nicht nur kennen, sondern auch verwenden), werden sie ihn möglicherweise nicht mehr als Helvetismus einstufen. Hier sollte in erster Linie die eigene Spracherfahrung, nicht die Kodifizierung in Wörterbüchern ausschlaggebend sein. Diese These konnte wegen der geringen Zahl der untersuchten Phraseme ebenfalls nicht statistisch untersucht werden. Ein Blick auf die relative Lage der Punkte in Abbildung 10 zeigt aber, dass sich zwischen den Gruppen der Phraseme, die gemäß Befragungsdaten als Helvetismen beziehungsweise Austro-, BavaroHelvetismen oder Alemannismen charakterisiert wurden, keine systematischen Unterschiede ausmachen lassen. $\mathrm{Zu}$ denjenigen Phrasemen, deren Einstufung als Helvetismus weniger häufig erfolgte, als dem Modell zufolge zu erwarten gewesen wäre (d.h. die Punkte liegen unterhalb der Geraden), zählten sowohl solche, die auf die Schweiz beschränkt sind, als auch solche mit grenzüberschreitender Verbreitung. ${ }^{32}$ Diesem kleinen Datensatz zufolge würde es für die Einstufung als Helvetismus prinzipiell keinen Unterschied machen, welche Reichweite ein Helvetismus im angrenzenden Ausland hat was ebenfalls anhand einer größeren Datengrundlage zu überprüfen ist.

Es mag deutlich geworden sein, dass die Klassifizierung eines Phrasems als Helvetismus durch eine ganze Reihe unterschiedlicher Kriterien beeinflusst werden kann. Neben diesen Einflussfaktoren ist auch auf die methodische Schwierigkeit der Erfassung des Helvetismenbewusstseins aufmerksam zu machen. Auch wenn im Fragebogen neben den Kategorien des Vorkommens „,nur innerhalb der Schweiz“ und ,auch außerhalb der Schweiz" auch die Antwortmöglichkeit „weiss nicht" vorgegeben war, ist nicht auszuschließen, dass diese Möglichkeit bei Unwissenheit selten angegeben und stattdessen geraten wurde. Die Kategorie „weiss nicht“ wurde von den Schweizer Informanten in $28 \%$ der Fälle, von Nicht-Schweizern in $47 \%$ der Fälle angekreuzt.

Für die in dieser Studie erfragten Phraseme ergab sich innerhalb der Schweiz eine Bewusstseinsrate von rund $50 \%$ (für die hier berücksichtigten vier Phraseme: 49\%, für alle elf: 46\%) bei einem durchschnittlichen Bekanntheitsgrad von 74\%. Mit anderen Worten: etwa jedem zweiten Sprechenden war der Helvetismenstatus eines Phrasems bewusst (im Einleitungstext zum Fragebogen wurde nur erwähnt, dass es um „Redewendungen“" ging, es wurde aber kein Bezug zu nationalen Varietäten hergestellt). Im Allgemeinen kann man wohl davon ausgehen, dass ein gewisser Bekanntheitsgrad die Voraussetzung für das Bewusstsein als Helvetismus darstellt. 


\section{Nationale wie areale Verbreitung phraseologischer Helvetismen}

\subsection{Räumliche Verbreitung und Ausdifferenzierung des Helvetismenbegriffs}

Die erste Frage an die empirischen Daten, die in Kapitel 3 gestellt wurde, bezog sich auf die räumliche Verbreitung von Phrasemen, die in den Wörterbüchern (Ammon et al. 2004; Dudenredaktion 2008; Meyer 2006) als Helvetismen kodifiziert sind. Die in Kapitel 5.2 dargestellten Ergebnisse haben gezeigt, dass diese Kodifizierungen nicht immer zuverlässig die tatsächliche Verbreitung der betreffenden Phraseme beschreibt. Exemplarisch wurde mit Hilfe empirischer Daten für sechs Phraseme gezeigt, dass die als Helvetismen klassifizierten Phraseme drei Gruppen angehören:

1. Phraseme, die nur in der Schweiz und hier überregional bekannt sind,

2. Phraseme, die in der Schweiz überregional, aber auch im angrenzenden österreichischen und/oder (einem Teil des) bundesdeutschen Raum(s) bekannt sind, und

3. Phraseme, die in der Schweiz nur regional bekannt sind.

Es konnte damit im phraseologischen Bereich gezeigt werden, dass sprachliche Einheiten und Merkmale sowohl für Nationen als auch grenzübergreifend für Areale charakteristisch sein können und damit für das Deutsche sowohl die Bezeichnungen Plurinationalität als auch Pluriarealität ihre Berechtigung haben. Dies beeinflusst den Status der Phraseme als Helvetismus nicht prinzipiell. Es wurde aber vorgeschlagen, bisherige terminologische Ungenauigkeiten zu konkretisieren, indem auch die areale Komponente bei der Bezeichnung der Phraseme berücksichtigt wird, und die Einheiten der 2. und 3. Gruppe nicht (nur) als Helvetismen zu bezeichnen, sondern ihre Verbreitung in der Bezeichnung genauer zu berücksichtigen, z.B. durch Benennungen wie Austro-Helvetismus, Alemannismus etc. Auf der Grundlage der geringen Phrasemzahl in dieser Studie ist selbstverständlich nicht quantifizierbar, ob nationale oder areale (Alemannisch, Bairisch) Grenzen den größeren Einfluss haben.

\subsection{Bewusstsein}

Die zweite Frage, die anhand der empirischen Daten untersucht werden sollte (siehe Kapitel 4), bezog sich auf das Sprachbewusstsein der Schweizer Informanten hinsichtlich ihrer Varietät, das auf der Ebene der einzelnen 
Helvetismen geprüft wurde. Es konnte gezeigt werden, dass die Einstufung durch eine Reihe verschiedener Faktoren beeinflusst werden kann. Ein zentraler Faktor, der 56\% der Variabilität in der Einstufung eines Phrasems erklärt, ist seine Bekanntheit. Ob und eine wie große Rolle andere Faktoren wie der Phrasemtyp (eigenständige Schweizer Einheiten, Schweizer Sonderphraseologie oder falsche Freunde), landestypische Hinweise und der Helvetismenstatus von Komponenten spielen, bleibt anhand umfangreicherer empirischer Untersuchungen zu erforschen.

Durchschnittlich hatten die in dieser Studie berücksichtigten phraseologischen Helvetismen, die von den Informanten räumlich zugeordnet wurden, einen Bewusstseinsgrad von 50\%, d.h. jedem zweiten Informanten war der Status eines Phrasems als Helvetismus bewusst. Ein Rückschluss vom Helvetismen- auf das Varietätenbewusstsein lässt sich insofern ziehen, als bei dem relativ geringen Bewusstsein auf der Phrasemebene davon auszugehen ist, dass das Bewusstsein der Schweizer Sprechenden um ihre nationalsprachliche Varietät nicht besonders stark ausgeprägt ist oder nach Scharloth (2004: o.S.) „das Plurizentrizitätskonzept [ist] noch auf dem Weg von den Köpfen der Linguisten in die Köpfe der Sprecher". ${ }^{33}$ Demnach erwüchse im Falle der Schweiz das Varietätenbewusstsein also nicht in erster Linie aus dem Bedürfnis des Sprechenden um nationale Identität und würde in seiner Folge vom Linguisten aufgenommen, sondern ginge tendenziell von der Linguistik - beispielsweise über Kodifzierungen - in Richtung der Sprechenden. Damit wäre das Kriterium des Sprechendenbewusstseins um die eigene Schweizer Varietät, das Koller (199:156, siehe Kapitel 2 und 4) als Voraussetzung für die Existenz einer nationalen Varietät fordert, nicht erfüllt. Das bisher begrenzte Bewusstsein der Sprechenden und seine Förderung von linguistischer Seite scheint meiner Meinung nach jedoch kein Ausschlusskriterium für das Bestehen einer nationalen Varietät zu sein. Vielmehr mag der Einzug von Schweizer Varianten in die Wörterbücher dazu führen, dass diese Varianten im nicht-dominanten Zentrum Schweiz eben nicht mehr als minderwertig (Scharloth 2004, 2005), sondern zunehmend als gleichberechtigt betrachtet werden. Die Kodifizierung dieser Varianten ermöglicht eine Entwicklung dahingehend, dass die Varianten des dominanten bundesdeutschen Zentrums keinen präskriptiven Charakter mehr haben und Kinder in Deutschschweizer Schulen lernen, dass Trottoir, Poulet und Velo keine Fehler, sondern gleichberechtigte Schweizer Besonderheiten neben dem bundesdeutschen Bürger-/Gehsteig, dem Hähnchen und dem Fahrrad darstellen. Gegen dieses bewusstseinsunterstützende oder z.T. -bildende Vorgehen von Linguistenseite, das das sprachliche (Selbst-)Bewusstsein der Sprechenden stärkt, ist nichts einzuwenden. 


\section{Anmerkungen}

Kontaktadresse: Britta.Juska-Bacher@unibas.ch

1. Mein Dank geht an die zwei anonymen Referent(inn)en für ihre sorgfältige Lektüre und konstruktiven Kritikpunkte zu einer früheren Version dieses Artikels.

2. Einen Überblick über die Herausbildung und Entwicklung des Konzepts Plurizentrik gibt Ammon (1995: 42-48).

3. $\mathrm{Zu}$ anderen plurizentrischen Sprachen siehe Clyne (1992).

4. Ein Beleg der Vermutung, dass die Gemeinsamkeiten zwischen Regionen unterschiedlicher nationaler Varietäten (z.B. im grenzüberschreitenden alemannischen oder bairischen Dialektraum) größer sind als die Gemeinsamkeiten innerhalb einer nationalen Varietät (z.B. im Schweizerdeutschen oder im österreichischen Standarddeutsch), müsste in den verschiedenen linguistischen Dimensionen wie Lexik, Semantik, Aussprache, Orthografie, Grammatik und Pragmatik quantifiziert werden.

5. Es kann sich dabei um Besonderheiten in der Schreibung, Aussprache, Grammatik oder Lexik der jeweiligen Varietät handeln.

6. In der lexikografischen Praxis werden beispielsweise im phraseologischen Bereich Helvetismen und Austriazismen als solche markiert, Teutonismen hingegen werden tendenziell eher als gemeindeutsch eingestuft.

7. Als weitere wichtige Bedingungen fordert Koller (1999: 156) ein zahlenmäßiges Gewicht der Schweizer Varianten und ihren Bezug auf zentrale Lebensbereiche der Sprechenden. Zu diesen Kriterien kann die in Kapitel 4.5 beschriebene Studie keine Hinweise liefern, da nur mit einer exemplarischen Auswahl von Phrasemen gearbeitet wird.

8. Burger (1995: 17) unterscheidet drei Typen: erstens „Sonderphraseologie“, d.h. Phraseme, die strukturelle Varianten zu bundesdeutschen und/oder österreichischen Einheiten darstellen, zweitens eigenständige Schweizer Phraseme (ohne Varianten in den anderen nationalen Varietäten) und drittens eine beschränkte Zahl von falschen Freunden.

9. In der Literatur wird daher auch zwischen sprachlichen Einheiten, die auf die Schweiz beschränkt sind, und solchen, die überwiegend, aber nicht ausschließlich in der Schweiz vorkommen, unterschieden. Haas (2000: 10) bezeichnet erstere als „absolute“ Helvetismen, Ammon (1995: 65) spricht von „spezifischen“ und Meyer (2001: 1191) von „arealgenauen“ Helvetismen. Einheiten, die nicht auf die Schweiz beschränkt sind, nennt Haas (2000: 10) „Frequenzhelvetismen“, Ammon (1995: 65) „unspezifische“ und Meyer (2001: 1191) „übergreifende“ Helvetismen, ohne dass dabei in der Bezeichnung die räumliche Erstreckung außerhalb der Schweiz konkretisiert würde. Die hier vorgeschlagenen Benennungen stellen eine Möglichkeit in dieser Richtung dar.

10. Meyer $(1989,2006)$ behilft sich daher mit der Kategorie „mundartnah“. 
11. Dies sowohl bei der Fragebogenerstellung (in der Diskussion mit den muttersprachlichen Studierenden) als auch von Seiten der Probanden, die gelegentlich Phraseme im Dialekt angaben: Beispielsweise wurde statt Jetzt ist genug Heu (dr)unten. auch Jetz isch gnueg heu dunde/donde/dunnä. genannt. Statt Jmd. kann mir in die Schuhe blasen. gaben Probanden auch an: Blas mer doch id Schue! und Du kasch mir in dschueh blose.

12. Dies verlangt indirekt auch Burger (1995: 14), wenn er für einen phraseologischen Helvetismus dessen Gebräuchlichkeit in der Sprachgemeinschaft fordert.

13. Zur Methode der Online-Befragung sowie zu ihren Vor- und Nachteilen siehe Juska-Bacher (2010). Die Probandengruppe ist nicht repräsentativ für die Grundgesamtheit der Sprechenden. Die bisher für Online-Erhebungen herausgestellten abweichenden Faktoren vom Bevölkerungsdurchschnitt hinsichtlich Alter, Geschlecht und Ausbildung (siehe Bandilla 1999: 15) wurden aber erfasst und gelten für alle hier untersuchten arealen Räume gleichermaßen, so dass eine Vergleichbarkeit wiederum gewährleistet ist. Hinsichtlich des Alters zeigte sich wie in verschiedenen anderen Erhebungen zur Bekanntheit von Phrasemen (z.B. Grzybek 1991: 247, Häcki Buhofer/Burger 1994: 15f, Juska-Bacher 2009: 171f), dass ältere Informanten von der Gesamtheit der abgefragten Phraseme durchschnittlich mehr kannten als jüngere. Bei einer Gruppierung der Informanten nach Alter in 10-Jahres-Schritten war ein Anstieg der durchschnittlichen Kenntnis von der Altersgruppe der 11- bis 20-Jährigen bis zur Gruppe der 61- bis 70-Jährigen, mit leichtem Abfall der durchschnittlichen Kenntnis der über 70-Jährigen zu erkennen. Im Hinblick auf die einzelnen Phraseme gab es aber durchaus solche, bei denen dieses Prinzip nicht galt (z.B. Zupf/züpf ich!) mit dem höchsten Bekanntheitsgrad in der Gruppe der 21- bis 30-Jährigen und dem geringsten in der Gruppe der über 70-jährigen Informanten und jmdm. die Stange halten in der Bedeutung „sich mit jmdm. messen“ mit dem größten Bekanntheitsgrad in der Gruppe der 21- bis 30-Jährigen und der 11- bis 20-Jährigen und einem deutlichen Abfall des Bekanntheitsgrades bis zur Unbekanntheit dieses Phrasems ab der Gruppe der 61-Jährigen. An der Befragung haben sich 545 Frauen und 432 Männer beteiligt (von fünf Informanten fehlen entsprechende Angaben), 435 der Informanten haben keine Universität besucht, 546 hingegen haben an einer Universität studiert (von einem Informanten fehlt diese Angabe).

14. Da davon ausgegangen wird, dass das Testen der Bekanntheit (durch Ergänzungstests oder Abfrage der Bedeutung) deutlich zuverlässiger ist als eine Selbsteinschätzung der Bekanntheit, der Verwendung oder der Verwendungshäufigkeit, wurde mit dem Testen der Bekanntheit gearbeitet.

15. Ihnen allen danke ich für ihr Interesse an diesem Thema und ihren Einsatz bei der Erarbeitung, Diskussion und Verbreitung des Fragebogens. Ebenso gedankt sei den 1000 Probanden, die an der Befragung teilgenommen haben.

16. Einleitungstext zu diesem Abschnitt:

„Bitte füllen Sie die Lücken mit demjenigen Wort, das Ihrer Meinung nach am ehesten in Ihrer Alltagssprache gebraucht wird. Dafür können Sie eine 
(oder mehrere) der Vorgaben wählen oder unter „Anderes, nämlich“ eine treffendere Möglichkeit angeben. Wenn Sie diese Wendung nicht kennen, bitte die letzte Zeile ankreuzen.“

Z.B. Frage 2:

„Wenn jemand von einer Situation überfordert und ratlos ist, steht er da wie der ... am/vorm Berg.
a. Bock
b. Esel
c. Ochs
d. Anderes, nämlich ...[Freifeld zur Eingabe]
e. Ich kenne diese Wendung nicht.“.

17. Einleitungstext zu diesem Abschnitt:

„Bitte geben Sie die Bedeutung der folgenden Wendungen an. Wählen Sie wieder eine (oder mehrere) der Vorgaben oder geben Sie eine treffendere Umschreibung an. Wenn Sie die Bedeutung nicht kennen, bitte einfach die letzte Zeile ankreuzen. Wir wüssten auch gern, ob es sich Ihrer Meinung nach um eine Redewendung handelt, die nur in der Schweiz oder auch ausserhalb der Schweiz gebräuchlich ist.“"

Z.B. Frage 17:

„das Feuer im Elsass sehen bedeutet:
a. starke Schmerzen haben
b. zu viel Phantasie haben
c. sehr gute Augen haben
d. Kenne ich nur in anderer Form, nämlich: ... [Freifeld zur Eingabe].“.

18. Ebenfalls zu Frage 17:

„Kommt diese Redewendung Ihrer Meinung nach

a. nur in der Schweiz vor?

b. auch ausserhalb der Schweiz vor?

c. weiss nicht.".

19. Es wurden nur Daten derjenigen Probanden verwendet, für die eine eindeutige Zuordnung zu einem der drei Vollzentren (oder Liechtenstein) möglich war (d.h. sie mussten den größten Teil ihres Lebens im betreffenden Land verbracht haben und dort auch sozialisiert worden sein).

20. Für die Kartenerstellung geht mein herzlicher Dank an Dr. Stefan Meier, Universität Basel.

21. Mehr als zwei Drittel der Informanten aus Baden-Württemberg hatten einen alemannischen Hintergrund, die übrigen machen diesbezüglich keine expliziten Angaben - es wird auch hier davon ausgegangen, dass der größere Teil aus den südlichen zwei Dritteln des Bundeslandes stammt und damit dem alemannischen Teil angehört.

22. 11 von 12 Informanten aus Bayern hatten einen bairischen Dialekthintergrund.

23. Österreich: 5 Probanden aus Vorarlberg, Oberösterreich, Kärnten und dem Burgenland; Liechtenstein: 7 Probanden. 
24. Die Nennformschreibung folgt Korhonen (2001).

25. Als ,(nahezu) unbekannt“ wurden in dieser Untersuchung Phraseme mit einem Bekanntheitsgrad von bis zu 20\% eingestuft. Dieser relativ hohe Schwellenwert sollte „zufällige“ arealfremde Kenntnis, Raten und Falschangaben weitgehend neutralisieren. Häufig liegt der tatsächliche Bekanntheitsgrad deutlich unter $20 \%$, im Fall von dastehen wie der Esel am Berg bspw. bei 5\%.

26. Siehe Fußnote 9.

27. In der Befragung wurden vereinzelt Formen wie das Feuer/die Sterne in Holland sehen angegeben. Inwieweit es sich hierbei um gängige Varianten handelt, müsste gesondert untersucht werden. In den dieser Untersuchung zugrunde gelegten Wörterbüchern sind diese Formen nicht aufgeführt. Im Schweizerischen Idiotikon (1881-, Bd. 1, Spalte 942) finden sich hingegen die Varianten Feuer z'Basel/im Schwarzwald/z'Bade mit der Bedeutungserläuterung ,i.S.v. in Folge eines Schlages oder Stosses an den Kopf subjektive Lichtempfindungen haben".

28. Ähnliches gilt auch für den phraseologischen falschen Freund jmdm. die Stange halten. In der Bedeutung „sich mit jmdm. messen“ war das Phrasem nur in der Westschweiz und hier zwischen 21 und 60\% der Informanten bekannt, in der östlichen Landeshälfte sowie in Österreich und Deutschland wurde nur die offensichtlich gemeindeutsche, ,jmdn. nicht im Stich lassen, ihm beistehen“ abgegeben.

29. Neben den in Abbildung 9 aufgeführten waren dies: Jetzt ist genug Heu (dr) unten. (,Jetzt ist aber genug.“); Das schleckt keine Geiss weg. (,Daran gibt es keinen Zweifel, es lässt sich nicht leugnen.“); Zupf/züpf dich. („Hau ab.“); jmdm. nimmt es den Ärmel rein (,Jmd. ist von einer Sache begeistert.“); jmdm. die Stange halten (,sich mit jmdm. messen“); mit abgesägten Hosen dastehen (,blossgestellt sein“); die Finken klopfen (,sich rasch und heimlich entfernen“).

30. Dieses Ergebnis widerspricht demjenigen von Di Paolo/Glaser (2006: 17), die bei einer Befragung von rund 300 Informanten feststellten, dass deutsche Teilnehmende sieben Helvetismen (Einzellexeme) häufiger als Helvetismen einstuften als Schweizer.

31. Berücksichtigt man die Ergebnisse verschiedener Studien, denen zufolge Informanten mit zunehmendem Alter mehr Phraseme kennen (siehe Fußnote 13), dann könnten ältere Informanten unter Umständen auch mehr Phraseme als Helvetismen einstufen, als es die Studierenden getan haben.

32. Jmd. kann mir in die Schuhe blasen., das neben der Schweiz auch in Österreich, Baden-Württemberg und Bayern als bekannt angegeben wurde (siehe Abbildung 7), beispielsweise lag recht genau auf der Regressionsgeraden und nicht, wie im Fall einer Bestätigung der o.g. Hypothese zu erwarten gewesen wäre, deutlich darunter.

33. Zu ähnlichem Ergebnis kommen auch Burger (1998) und Scharloth (2004 und 2005). 


\section{Bibliografie}

Ammon, Ulrich. 1986. Explikation der Begriffe ,Standardvarietät' und ,Standardsprache' auf normtheoretischer Grundlage. In Günter Holtus, Edgar Radtke (Hrsg.), Sprachlicher Substandard, 1-63. Tübingen: Niemeyer.

Ammon, Ulrich. 1995. Die deutsche Sprache in Deutschland, Österreich und der Schweiz: Das Problem der nationalen Varietäten. Berlin/New York: Walter de Gruyter.

Ammon, Ulrich, Hans Bickel, Jakob Ebner, Ruth Esterhammer, Markus Gasser, Lorenz Hofer, Birte Kellermeier-Rehbein, Heinrich Löffler, Doris Mangott, Hans Moser, Robert Schläpfer, Michael Schloßmacher, Regula Schmidlin \& Günter Vallaster. 2004. Variantenwörterbuch des Deutschen: Die Standardsprache in Österreich, der Schweiz und Deutschland sowie in Liechtenstein, Luxemburg, Ostbelgien und Südtirol. Berlin: Walter de Gruyter.

Bandilla, Wolfgang. 1999. WWW-Umfragen - Eine alternative Datenerhebungstechnik für die empirische Sozialforschung? In Bernad Batinic, Andreas Werner, Lorenz Gräf \& Wolfgang Bandilla (Hrsg.), Online Research: Methoden, Anwendungen und Ergebnisse, 9-19. Göttingen/Bern/Toronto/Seattle: Hogrefe.

Bickel, Hans. 2006. Das Internet als linguistisches Korpus. Linguistik online 28(3). 71-83.

Burger, Harald. 1995. Helvetismen in der Phraseologie. In Heinrich Löffler (Hrsg.), Alemannische Dialektforschung: Bilanz und Perspektiven, 13-25. Tübingen, Basel: Francke.

Burger, Harald. 1996. Zur Phraseologie des Schweizerdeutschen. In Jarmo Korhonen (Hrsg.), Studien zur Phraseologie des Deutschen und des Finnischen II, 461-488. Bochum: Brockmeyer.

Burger, Harald. 1998. Helvetismen in der Phraseologie - Vorkommen und stilistische Funktionen. In Dietrich Hartmann (Hrsg.), „Das geht auf keine Kuhhaut". Arbeitsfelder der Phraseologie: Akten des Westfälischen Arbeitskreises Phraseologie/Parömiologie 1996 (Bochum), 49-80. Bochum: Brockmeyer.

Burger, Harald. 2007. Phraseologie: Eine Einführung am Beispiel des Deutschen. Berlin: Erich Schmidt Verlag.

Clyne, Michael George. 1984. Language and society in the German-speaking countries. Cambridge: Cambridge University Press.

Clyne, Michael George. 1992. Pluricentric languages: Differing norms in different nations. Berlin: Mouton de Gruyter.

Di Paolo, Maria Concetta \& Elvira Glaser. 2006. Wie lassen sich Helvetismen erkennen? In Christa Dürscheid \& Martin Businger (Hrsg.), Schweizer Standarddeutsch: Beiträge zur Varietätenlinguistik, 11-22. Tübingen: Gunter Narr.

Dudenredaktion (Hrsg.). 1984. Die Grammatik. Bearb. von Günter Drosdowski. Mannheim etc.: Dudenverlag. Bd. 4.

Dudenredaktion (Hrsg.). 1990. Aussprachewörterbuch: Wörterbuch der deutschen Standardaussprache. Bearb. von Max Mangold. Mannheim etc.: Dudenverlag. Bd. 6. 
Dudenredaktion (Hrsg.). 1991. Rechtschreibung der deutschen Sprache: auf der Grundlage der amtlichen Rechtschreibregeln. Mannheim etc.: Dudenverlag. Bd. 1.

Dudenredaktion (Hrsg.). 1992. Redewendungen und sprichwörtliche Redensarten: Wörterbuch der deutschen Idiomatik. Bearb. von Günther Drosdowski und Werner Scholze-Stubenrecht. Mannheim etc.: Dudenverlag. Bd. 11.

Dudenredaktion (Hrsg.). 2008. Redewendungen. Bearb. von Werner ScholzeStubenrecht. Mannheim etc.: Dudenverlag. Bd. 11.

Ebner, Jakob. 2009. Wie sagt man in Österreich? Wörterbuch des österreichischen Deutsch. Mannheim etc.: Dudenverlag.

Földes, Csaba. 1992. Zu den österreichischen Besonderheiten der deutschen Phraseologie. In Csaba Földes (Hrsg.), Deutsche Phraseologie in Sprachsystem und Sprachverwendung, 9-24, Wien: Praesens.

Földes, Csaba. 1996. Deutsche Phraseologie kontrastiv - intra- und interlingale Zugänge. Heidelberg: Groos.

Grzybek, Peter. 1991. Sinkendes Kulturgut? Eine empirische Pilotstudie zur Bekanntheit deutscher Sprichwörter. Wirkendes Wort 2. 239-264.

Haas, Walter. 1982. Die deutschsprachige Schweiz. In Jachen C Arquint, Iso Camartin, Walter Haas, Pierre Knecht, Ottavio Lurati, Florentin Lutz \& Robert Schläpfer, Die viersprachige Schweiz,71-160. Zürich, Köln: Benziger.

Haas, Walter. 2000. Die deutschsprachige Schweiz. In Hans Bickel \& Robert Schläpfer (Hrsg.), Die viersprachige Schweiz, 57-138. Aarau et al.: Sauerländer.

Häcki Buhofer, Annelies. 1998. Kenntnis- und Gebrauchsunterschiede bei Phraseologismen des Binnendeutschen, des Schweizerhochdeutschen und des Schweizerdeutschen. In Wolfgang Eismann (Hrsg.), Europhras 95 - Europäische Phraseologie im Vergleich: Gemeinsames Erbe und kulturelle Vielfalt, 295-313. Bochum: Norbert Brockmeyer.

Häcki Buhofer, Annelies \& Harald Burger. 1994. Phraseologismen im Urteil von Sprecherinnen und Sprechern. In Barbara Sandig (Hrsg.), EUROPHRAS 92. Tendenzen der Phraseologieforschung, 1-33. Bochum: Brockmeyer.

Hofer, Lorenz. 2003. Phraseologismen im Wörterbuch der nationalen Varianten der deutschen Standardsprache. In Harald Burger, Annelies Häcki Buhofer \& Gertrud Gréciano (Hrsg.), Flut von Texten - Vielfalt der Kulturen. Ascona 2001 zur Methodologie und Kulturspezifik der Phraseologie, 479-490. Baltmannsweiler: Schneider.

Juska-Bacher, Britta. 2009. Empirisch-kontrastive Phraseologie: Am Beispiel der Bekanntheit von Bruegels Niederländischen „Sprichwörtern“ im Niederländischen, Deutschen und Schwedischen. Baltmannsweiler: Schneider.

Juska-Bacher, Britta. 2010. SDS-Exploratoren und Online-Befragung - Lässt sich im Methodenmix ein Wandel in der Schweizer Dialektlandschaft nachweisen? In Helen Christen, Sibylle Germann, Nadia Montefiori, Walter Haas \& Hans Ruef (Hrsg.), Dialektologie: Wege in die Zukunft (Beihefte zur Zeitschrift für Dialektologie und Linguistik), 279-293. Stuttgart: Steiner Verlag. 
Koller, Werner. 1999. Nationale Sprach(en)kultur der Schweiz und die Frage der „nationalen Varietäten des Deutschen“. In Andreas Gardt, Ulrike Haß-Zumkehr \& Thorsten Roelcke (Hrsg.), Sprachgeschichte als Kulturgeschichte, 133-170. Berlin/New York: Walter de Gruyter.

Meyer, Kurt. 1989. Wie sagt man in der Schweiz? Wörterbuch der schweizerischen Besonderheiten. Mannheim: Bibliographisches Institut.

Meyer, Kurt. 2001. Die lexikalische Situation des Standarddeutschen in der Schweiz. In D. Alan Cruse, Franz Hundsnurscher, Michael Job \& Peter Rolf Lutzeier (Hrsg.), An international handbook on the nature and structure of words and vocabularies / Ein internationales Handbuch zur Natur und Struktur von Wörtern und Wortschätzen, 1189-1196. Berlin/New York: Walter de Gruyter.

Meyer, Kurt. 2006. Schweizer Wörterbuch: So sagen wir in der Schweiz. Frauenfeld/Stuttgart/Wien: Huber.

von Peter Polenz. 1999. Deutsch als plurinationale Sprache im postnationalistischen Zeitalter. In Andreas Gardt, Ulrike Haß-Zumkehr \& Thorsten Roelcke (Hrsg.), Sprachgeschichte als Kulturgeschichte, 115-132. Berlin/New York: Walter de Gruyter.

Reiffenstein, Ingo. 2001. Das Problem der nationalen Varietäten. Rezensionsaufsatz zu Ulrich Ammon: Die deutsche Sprache in Deutschland, Österreich und der Schweiz. Das Problem der nationalen Varietäten, Berlin, New York 1995. ZfdPh 120. 78-89.

Scharloth, Joachim. 2004. Zwischen Fremdsprache und nationaler Varietät: Untersuchungen zum Plurizentrizitätsbewusstsein der Deutschschweizer. In Rudolf Muhr (Hrsg.), Standardvariationen und Sprachideologien in verschiedenen Sprachkulturen der Welt / Standard Variations and Language Ideologies in Different Language Cultures around the World, Frankfurt am Main etc.: Lang. [Zugriff am 20 112010 unter http://www.inst.at/trans/15Nr/06_1/scharloth15.htm]

Scharloth, Joachim. 2005. Asymmetrische Plurizentrizität und Sprachbewusstsein. Einstellungen der Deutschschweizer zum Standarddeutschen. Zeitschrift für Germanistische Linguistik 33(2). 236-267.

Scheuringer, Hermann. 1990. Sprachentwicklung in Bayern und Österreich. Eine Analyse des Substandardverhaltens der Städte Braunau am Inn (Österreich) und Simbach am Inn (Bayern) und ihres Umlandes. Hamburg: Helmut Buske.

Scheuringer, Hermann. 1997. Sprachvarietäten in Österreich. In Gerhard Stickel (Hrsg.), Varietäten des Deutschen: Regional- und Umgangssprachen, 332-345. Berlin/New York: Walter de Gruyter.

Schifferle, Hans-Peter. 1995. Dialektstrukturen in Grenzlandschaften. Untersuchungen zum Mundartwandel im nordöstlichen Aargau und im benachbarten südbadischen Raum Waldshut. Bern etc.: Peter Lang.

Schmidlin, Regula. 2004. Nationale Varianten standarddeutscher Phraseologismen. In Christine Palm Meister (Hrsg.): EUROPHRAS 2000: Internationale Tagung zur Phraseologie vom 15. - 18. Juni 2000 in Aske/Schweden, 435-447. Tübingen: Stauffenburg. 
Schmidlin, Regula. 2004a. Lexikographische Probleme bei phraseologischen Varianten. In Csaba Földes \& Jan Wirrer (Hrsg.), Phraseologismen als Gegenstand sprach- und kulturwissenschaftlicher Forschung. Akten der Europäischen Gesellschaft für Phraseologie (EUROPHRAS) und des Westfälischen Arbeitskreises „Phraseologie/Parömiologie“ (Loccum 2002), 377-391. Baltmannsweiler: Schneider.

Schmidlin, Regula. 2007. Phraseological expressions in German standard varieties. In Harald Burger, Dmitrij Dobrovol'skij, Peter Kühn \& Neal R. Norrick (Hrsg.), Phraseologie. Phraseology, 551-562. Berlin, New York: Walter de Gruyter.

Schweizer Schülerduden. 1980, 1976. Wabern: Büchler. 2 Bde.

Seidelmann, Erich. 1983. Die Stadt Konstanz und die Sprachlandschaft am Bodensee. In Hugo Steger, Eugen Gabriel \& Volker Schupp (Hrsg.), Forschungsbericht Südwestdeutscher Sprachatlas, 156-234. Marburg: Elwert.

Schweizerisches Idiotikon (1881-). Frauenfeld: Huber.

Siebs, Theodor. 1969. Deutsche Aussprache: Reine und gemäßigte Hochlautung mit Aussprachewörterbuch. Hrsg. von Helmut de Boor, Hugo Moser \& Christian Winkler. Berlin: Walter de Gruyter. 


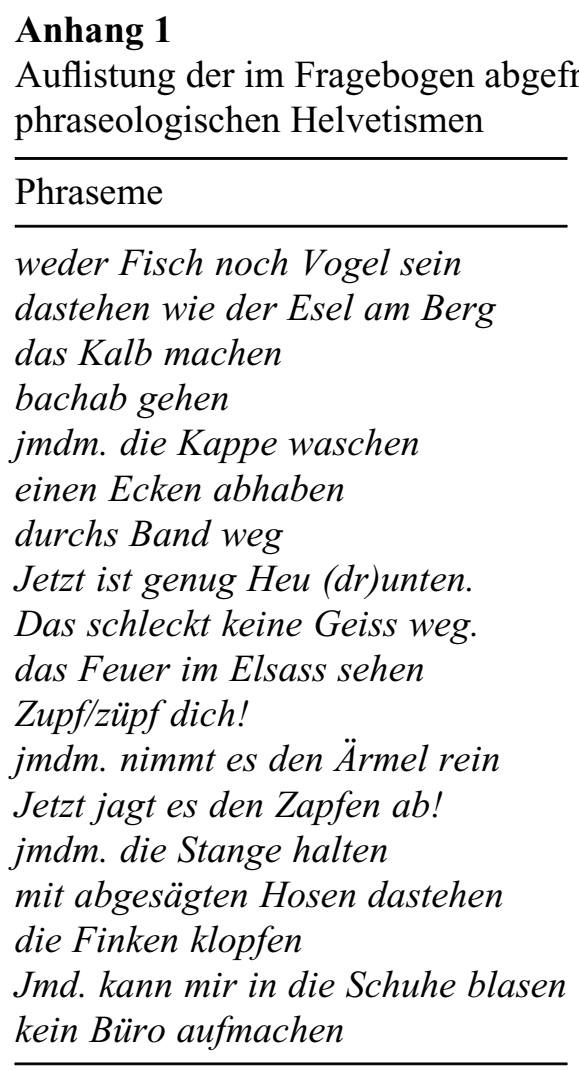




\section{Anhang 2}

Die Herkunft der Informanten nach Kantonen bzw. Bundesländern

\begin{tabular}{|c|c|c|c|}
\hline Land & Verwaltungseinheit & Anzahl & gesamt \\
\hline \multirow[t]{20}{*}{ Schweiz } & Aargau & 100 & \\
\hline & Appenzell Ausserrhoden & 8 & \\
\hline & Appenzell Innerrhoden & 1 & \\
\hline & Basel-Landschaft & 95 & \\
\hline & Basel-Stadt & 126 & \\
\hline & Bern & 84 & \\
\hline & Freiburg & 7 & \\
\hline & Glarus & 1 & \\
\hline & Graubünden & 10 & \\
\hline & Luzern & 38 & \\
\hline & Nidwalden & 2 & \\
\hline & Schaffhausen & 9 & \\
\hline & Schwyz & 24 & \\
\hline & Solothurn & 57 & \\
\hline & St. Gallen & 104 & \\
\hline & Thurgau & 17 & \\
\hline & Uri & 1 & \\
\hline & Wallis & 14 & \\
\hline & Zug & 17 & \\
\hline & Zürich & 148 & 863 \\
\hline \multirow[t]{3}{*}{ Deutschland } & Baden-Württemberg & 45 & \\
\hline & Bayern & 12 & \\
\hline & übrige Bundesländer & 49 & 106 \\
\hline Österreich & & & 5 \\
\hline Liechtenstein & & & 7 \\
\hline
\end{tabular}

